TRANSACTIONS OF THE

AMERICAN MATHEMATICAL SOCIETY

Volume 354, Number 1, Pages 123-149

S 0002-9947(01)02817-3

Article electronically published on June 6, 2001

\title{
ON ARITHMETIC MACAULAYFICATION OF NOETHERIAN RINGS
}

\author{
TAKESI KAWASAKI
}

\begin{abstract}
The Rees algebra is the homogeneous coordinate ring of a blowingup. The present paper gives a necessary and sufficient condition for a Noetherian local ring to have a Cohen-Macaulay Rees algebra: A Noetherian local ring has a Cohen-Macaulay Rees algebra if and only if it is unmixed and all the formal fibers of it are Cohen-Macaulay. As a consequence of it, we characterize a homomorphic image of a Cohen-Macaulay local ring. For non-local rings, this paper gives only a sufficient condition. By using it, however, we obtain the affirmative answer to Sharp's conjecture. That is, a Noetherian ring having a dualizing complex is a homomorphic image of a finite-dimensional Gorenstein ring.
\end{abstract}

\section{INTRODUCTION}

Let $A$ be a commutative ring with identity and $\mathfrak{b}$ an ideal in $A$. The Rees algebra of $\mathfrak{b}$ is the graded ring

$$
R(\mathfrak{b})=\bigoplus_{n \geq 0}(\mathfrak{b} T)^{n}
$$

where $T$ is an indeterminate. We often regard $R(\mathfrak{b})$ as an $A$-subalgebra $A[\mathfrak{b} T]$ of the polynomial ring $A[T]$. The Rees algebra is an important object of Algebraic Geometry and Commutative Algebra because the canonical morphism Proj $R(\mathfrak{b}) \rightarrow$ $\operatorname{Spec} A$ is the blowing-up of $\operatorname{Spec} A$ along the closed subscheme $\operatorname{Spec} A / \mathfrak{b}$.

In the present paper, we consider the existence of Cohen-Macaulay Rees algebras. A Rees algebra $R(\mathfrak{b})$ is said to be an arithmetic Macaulayfication of $A$ if it is Cohen-Macaulay and $\mathfrak{b}$ is of positive height. The main theorem of this paper is the following.

Theorem 1.1. Let $A$ be a Noetherian local ring of positive dimension. Then the following statements are equivalent:

(A) A has an arithmetic Macaulayfication;

(B) $A$ is unmixed and all the formal fibers of $A$ are Cohen-Macaulay.

Here a Noetherian local ring $A$ is said to be unmixed if $\operatorname{dim} \hat{A} / \mathfrak{p}=\operatorname{dim} \hat{A}$ for every associated prime $\mathfrak{p}$ of the completion $\hat{A}$. The formal fibers of $A$ are the fiber rings of the natural homomorphism $A \rightarrow \hat{A}$.

Received by the editors February 15, 2000.

1991 Mathematics Subject Classification. Primary 13A30; Secondary 13D45, 13H10.

Key words and phrases. Arithmetic Macaulayfication, Cohen-Macaulay rings, dualizing complex, excellent rings, formal fibers, local cohomology, Macaulayfication, Rees algebra.

The author is partially supported by Grant-in-Aid for Co-Operative Research. 
The studies in the Cohen-Macaulay property of Rees algebras started from Barshay's paper [5]. He gave the defining ideal of $R(\mathfrak{b})$ and its free resolution if $\mathfrak{b}$ is generated by a regular sequence. He also showed that $R(\mathfrak{b})$ is Cohen-Macaulay if $A$ is also and if $\mathfrak{b}$ is generated by a regular sequence. Around 1980, Goto and Shimoda studied several properties of $R(\mathfrak{b})$ in the case where $A$ is a Buchsbaum local ring and $\mathfrak{b}$ a parameter ideal. See [9], [10, [11, and [31. Summarizing these investigations, Goto and Yamagishi [12] established the theory of unconditioned strong $d$-sequences. Their theory contains the existence of an arithmetic Macaulayfication in the case where $A$ is unmixed and Spec $\hat{A}$ is Cohen-Macaulay except for the closed point. See also Brodmann [7] and Schenzel [27]. Recently Kurano [19] proved that a Noetherian local ring $A$ containing a finite field has an arithmetic Macaulayfication if the non- $F$-rational locus of $A$ is of dimension 1 . Independently this was also done by Aberbach 11. Motivated by Kurano's work, the author [18] also gave some sufficient conditions for $A$ to have an arithmetic Macaulayfication. Theorem 1.1 gives a necessary and sufficient condition for an arithmetic Macaulayfication to exist.

If the Rees algebra $R(\mathfrak{b})$ is a Cohen-Macaulay ring, then the projective scheme $\operatorname{Proj} R(\mathfrak{b})$ is Cohen-Macaulay. However, the converse is not true in general. The author [17] gave an ideal $\mathfrak{b}$ such that $\operatorname{Proj} R(\mathfrak{b})$ is a Cohen-Macaulay scheme for fairly general Noetherian local rings. Theorem 1.1 gives another proof of the result in 17 .

In our arithmetic Macaulayfication $R(\mathfrak{b})$, the ideal $\mathfrak{b}$ is generated by monomials of a certain system of parameters, named a $p$-standard system of parameters. Sections 2 and 3 are devoted to discussing the existence and properties of a $p$-standard system of parameters. Theorems 2.5 and 3.6 are improvements of Theorems 2.7 and 3.1 of [17, respectively. We give a proof of Theorem[1.1] in Section 4. In our proof the theory of multigraded Rees algebras, which was introduced by Herrmann, Hyry, and Ribbe [15, plays a key role. Our ideal $\mathfrak{b}$ is very complicated. However, their theory makes the proof of Theorem 1.1 simple.

In section 5 we give a consequence of Theorem 1.1.

Corollary 1.2. A Noetherian local ring is a homomorphic image of a CohenMacaulay local ring if and only if it is universally catenary and all the formal fibers of it are Cohen-Macaulay. An excellent local ring is a homomorphic image of a Cohen-Macaulay excellent local ring.

However, there exists no analogy with the Gorenstein property. In fact, Ogoma 22, Example 1] gave an example of an acceptable local ring which is not a homomorphic image of a Gorenstein ring.

For non-local rings, this paper gives only a sufficient condition for an arithmetic Macaulayfication to exist.

Theorem 1.3. Let $B$ be a Noetherian ring possessing a dualizing complex. If the codimension function is a constant on the associated primes of $B$, then $B$ has an arithmetic Macaulayfication.

We refer the readers to Section 5 for the definition of the codimension function.

By using Theorem 1.3, we give an affirmative answer to Sharp's conjecture 30 . Conjecture 4.4].

Corollary 1.4. A Noetherian ring has a dualizing complex if and only if it is a homomorphic image of a finite-dimensional Gorenstein ring. 
This is a simple criterion for a dualizing complex to exist. Several authors gave partial answers. See [2], [3], [4], [22], and [23]. We give proofs of Theorem [1.3 and Corollary 1.4 in Section 6 ,

Throughout this paper, $A$ denotes a Noetherian local ring with maximal ideal $\mathfrak{m}$. We assume that the dimension of $A$ is positive. We refer the reader to [13, [14, and 20], for unexplained terminology.

\section{A p-STANDARD SYSTEM OF PARAMETERS, I}

In this section, we give the definition of a $p$-standard system of parameters and discuss the existence of it. For a finitely generated $A$-module $M$, let $\mathfrak{a}^{p}(M)$ denote the annihilator of the $p$ th local cohomology module $H_{\mathfrak{m}}^{p}(M)$ of $M$ and let $\mathfrak{a}(M)=\prod_{p<\operatorname{dim} M} \mathfrak{a}^{p}(M)$.

Definition 2.1. Let $M$ be a finitely generated $A$-module of dimension $d>0$, $x_{1}, \ldots, x_{d}$ a system of parameters for $M$ and $s$ an integer such that $0 \leq s<d$. We say that $x_{1}, \ldots, x_{d}$ is a $p$-standard system of parameters of type $s$ for $M$ if

(1) $x_{s+1}, \ldots, x_{d} \in \mathfrak{a}(M)$;

(2) $x_{i} \in \mathfrak{a}\left(M /\left(x_{i+1}, \ldots, x_{d}\right) M\right)$ for $1 \leq i \leq s$.

This notion was given by N. T. Cuong [8]. He showed that there exists a $p$ standard system of parameters of type $d-1$ for $M$ whenever $A$ possesses a dualizing complex. We improve his result. For a finitely generated $A$-module $M$, let $\operatorname{NCM}(M)$ denote the non-Cohen-Macaulay locus of $M$, that is, $\operatorname{NCM}(M)=\left\{\mathfrak{p} \in \operatorname{Spec} A \mid M_{\mathfrak{p}}\right.$ is not a Cohen-Macaulay $A_{\mathfrak{p}}$-module\}. By modifying the proof of [29, Theorem 3.3], we obtain the following lemma.

Lemma 2.2. Let $B$ and $C$ be Noetherian rings and $B \rightarrow C$ a faithfully flat ring homomorphism. We assume that $C_{\mathfrak{p}} / \mathfrak{p} C_{\mathfrak{p}}$ is a Cohen-Macaulay ring for every prime ideal $\mathfrak{p}$ in $B$. Let $M$ be a finitely generated $B$-module. If there exists an ideal $\mathfrak{c}$ in $C$ such that $\operatorname{NCM}\left(M \otimes_{B} C\right)=V(\mathfrak{c})$, then $\operatorname{NCM}(M)=V(\mathfrak{c} \cap B)$.

We need the following propositions to choose a $p$-standard system of parameters.

Proposition 2.3. Assume that $A$ is universally catenary and that all the formal fibers of $A$ are Cohen-Macaulay. Let $M$ be a finitely generated $A$-module of dimension $d>0$. If $M$ is equidimensional, then $\operatorname{NCM}(M)=V(\mathfrak{a}(M))$. In particular, $\operatorname{dim} A / \mathfrak{a}(M)<d$.

Proof. If $A$ has a dualizing complex, then the assertion was given by Schenzel 26 . p. 52]. Assume that $A$ has no dualizing complex. The completion $\hat{A}$ of $A$ has a dualizing complex and is a faithfully flat $A$-algebra. Since $A$ is formally catenary, $M \otimes \hat{A}$ is also equidimensional. Therefore the non-Cohen-Macaulay locus of $M \otimes \hat{A}$ is

$$
V(\mathfrak{a}(M \otimes \hat{A}))=V\left(\mathfrak{a}^{0}(M \otimes \hat{A}) \cap \cdots \cap \mathfrak{a}^{d-1}(M \otimes \hat{A})\right) .
$$

By using Lemma 2.2 we find that the non-Cohen-Macaulay locus of $M$ is

$$
V\left(\mathfrak{a}^{0}(M \otimes \hat{A}) \cap \cdots \cap \mathfrak{a}^{d-1}(M \otimes \hat{A}) \cap A\right)=V\left(\mathfrak{a}^{0}(M) \cap \cdots \cap \mathfrak{a}^{d-1}(M)\right) .
$$

The right-hand side of the equation above is equal to $V(\mathfrak{a}(M))$. Since $\operatorname{NCM}(M)$ contains no minimal prime of $M, \operatorname{dim} A / \mathfrak{a}(M)=\operatorname{dim} \mathrm{NCM}(M)<d$. 
Corollary 2.4. Assume that $A$ is universally catenary and that all the formal fibers of $A$ are Cohen-Macaulay. Let $M$ be a finitely generated $A$-module of dimension $d>$ 0 . If $\operatorname{dim} A / \mathfrak{p}=d$ for every associated prime ideal $\mathfrak{p}$ of $M$, then $\operatorname{dim} A / \mathfrak{a}(M)<d-1$.

Proof. Let $\mathfrak{p}$ be a prime ideal of $A$ such that $\operatorname{dim} A / \mathfrak{p}=d-1$ and $M_{\mathfrak{p}} \neq 0$. Then the one-dimensional $A_{\mathfrak{p}}$-module $M_{\mathfrak{p}}$ is Cohen-Macaulay because $M_{\mathfrak{p}}$ has no embedded prime. Therefore $\operatorname{dim} A / \mathfrak{a}(M)=\operatorname{dim} \operatorname{NCM}(M)<d-1$.

The following theorem assures us of the existence of the $p$-standard system of parameters.

Theorem 2.5. Assume that $A$ is universally catenary and that all the formal fibers of $A$ are Cohen-Macaulay. Let $M$ be a finitely generated $A$-module of dimension $d>$ 0 . If $M$ is equidimensional and $s$ an integer such that $\operatorname{dim} A / \mathfrak{a}(M) \leq s<d$, then there exists a p-standard system of parameters of type $s$ for $M$.

Proof. Since $d-\operatorname{dim} A / \mathfrak{a}(M) \geq d-s$, there exist $d-s$ elements $x_{s+1}, \ldots, x_{d}$ in $\mathfrak{a}(M)$ such that $\operatorname{dim} M /\left(x_{s+1}, \ldots, x_{d}\right) M=s$. If elements $x_{i+1}, \ldots, x_{d}$ in $A$ such that $\operatorname{dim} M /\left(x_{i+1}, \ldots, x_{d}\right) M=i$ are given, then $M /\left(x_{i+1}, \ldots, x_{d}\right) M$ is also equidimensional. Therefore $\operatorname{dim} A / \mathfrak{a}\left(M /\left(x_{i+1}, \ldots, x_{d}\right) M\right)<i$ and hence there exists an element $x_{i}$ in $\mathfrak{a}\left(M /\left(x_{i+1}, \ldots, x_{d}\right) M\right)$ such that $\operatorname{dim} M /\left(x_{i}, \ldots, x_{d}\right) M=i-1$.

\section{A $p$-STANDARD SYSTEM OF PARAMETERS, II}

In this section, we give some properties of a $p$-standard system of parameters. First we recall the definition of $d$-sequences and the one of unconditioned strong $d$-sequences.

Definition 3.1. Let $M$ be an $A$-module. A sequence $x_{1}, \ldots, x_{d}$ of elements in $A$ is said to be a $d$-sequence on $M$ if

$$
\left(x_{1}, \ldots, x_{i-1}\right) M: x_{i} x_{j}=\left(x_{1}, \ldots, x_{i-1}\right) M: x_{j}
$$

for any $1 \leq i \leq j \leq d$. Here we set $\left(x_{1}, \ldots, x_{i-1}\right)=(0)$ if $i=1$.

A sequence $x_{1}, \ldots, x_{d}$ of elements in $A$ is said to be an unconditioned strong $d$-sequence (for short, a u.s.d-sequence) on $M$ if $x_{1}^{n_{1}}, \ldots, x_{d}^{n_{d}}$ is a $d$-sequence on $M$ for any positive integers $n_{1}, \ldots, n_{d}$ and in any order.

The following is one of the important properties of $d$-sequences. It was first given by Goto and Shimoda [11, Lemma 4.2] for the system of parameters for a Buchsbaum local ring, which is a typical example of $d$-sequences.

Proposition 3.2 ([12, Theorem 1.3]). Let $M$ be an A-module and $x_{1}, \ldots, x_{d}$ a $d$-sequence on $M$. If we put $\mathfrak{q}=\left(x_{1}, \ldots, x_{d}\right)$, then

$$
\left(x_{1}, \ldots, x_{i-1}\right) M: x_{i} \cap \mathfrak{q}^{n} M=\left(x_{1}, \ldots, x_{i-1}\right) \mathfrak{q}^{n-1} M
$$

for any $n>0$ and $1 \leq i \leq d$.

A $p$-standard system of parameters has several nice properties. The following two properties are given in [17.

Proposition 3.3 ([17, Proposition 2.8]). Let $M$ be a finitely generated A-module of dimension $d>0$ and $x_{1}, \ldots, x_{d}$ a p-standard system of parameters of type $s$ for $M$. Then $x_{s+1}, \ldots, x_{d}$ is a u.s.d-sequence on $M /\left(y_{1}, \ldots, y_{u}\right) M$ where $y_{1}, \ldots$, $y_{u}$ is a subsystem of parameters for $M /\left(x_{s+1}, \ldots, x_{d}\right) M$. 
Proposition 3.4 ([17] Theorem 2.9]). Let $M$ be a finitely generated A-module of dimension $d>0, x_{1}, \ldots, x_{d}$ a p-standard system of parameters of type $s$ for $M$, and $y_{1}, \ldots, y_{u}$ a subsystem of parameters for $M /\left(x_{i}, \ldots, x_{d}\right) M$ where $2 \leq i \leq d$ and $1 \leq u<i$. If $y_{u} \in \mathfrak{a}(M)$ or $y_{u} \in \mathfrak{a}\left(M /\left(x_{i}, \ldots, x_{d}\right) M\right)$, then

$$
\left(y_{1}, \ldots, y_{v-1},\left\{x_{\lambda} \mid \lambda \in \Lambda\right\}\right) M: y_{v} y_{u}=\left(y_{1}, \ldots, y_{v-1},\left\{x_{\lambda} \mid \lambda \in \Lambda\right\}\right) M: y_{u}
$$

for any $1 \leq v \leq u$ and $\Lambda \subseteq\{i, \ldots, d\}$.

The next proposition is not in [17] but we need it to prove Theorem 1.1. The author is inspired by [8, Theorem 2.6].

Proposition 3.5. Let $M$ be a finitely generated A-module of dimension $d>0$, $x_{1}, \ldots, x_{d}$ a p-standard system of parameters of type $s$ for $M$ and $y_{1}, \ldots, y_{u}$ a subsystem of parameters for $M /\left(x_{i}, \ldots, x_{d}\right) M$ where $1 \leq i \leq d$ and $1 \leq u<i$. Then $x_{i}, \ldots, x_{j}$ is a $d$-sequence on $M /\left(y_{1}, \ldots, y_{u}, x_{j+1}, \ldots, x_{d}\right) M$ for any $i \leq j \leq d$.

Proof. Let $i \leq l \leq j$ be an integer. By applying Proposition 3.4 to a subsystem of parameters $y_{1}, \ldots, y_{u}, x_{i}, \ldots, x_{l}$ for $M /\left(x_{l+1}, \ldots, x_{d}\right) M$ and a subset $\{j+1, \ldots, d\}$ of $\{l+1, \ldots, d\}$, we obtain

$$
\begin{aligned}
& \left(y_{1}, \ldots, y_{u}, x_{i}, \ldots, x_{k-1}, x_{j+1}, \ldots, x_{d}\right) M: x_{k} x_{l} \\
& \quad=\left(y_{1}, \ldots, y_{u}, x_{i}, \ldots, x_{k-1}, x_{j+1}, \ldots, x_{d}\right) M: x_{l}
\end{aligned}
$$

for any $i \leq k \leq l$.

The following theorem and corollaries are improvements of Theorem 3.1, Corollaries 3.2 and 3.3 of [17, respectively. The old theorems require that all $n_{i}, \ldots, n_{j}$ are positive but new ones require only that all $n_{i}, \ldots, n_{j}$ are nonnegative.

Theorem 3.6. Let $M$ be a finitely generated A-module of dimension $d>0$ and $x_{1}, \ldots, x_{d}$ a p-standard system of parameters of type $s$ for $M$. We put $\mathfrak{q}_{i}=$ $\left(x_{i}, \ldots, x_{d}\right)$ for all $1 \leq i \leq d$. Then, for any integers $1 \leq i \leq j \leq d$ and $n_{i}, \ldots$, $n_{j} \geq 0$, the following statements hold:

$\left(A_{i j}\right)$ If $y_{1}, \ldots, y_{u}$ is a subsystem of parameters for $M / \mathfrak{q}_{i} M$ and if $n_{k}>0$ for some integer $i \leq k \leq j$, then

$$
\begin{gathered}
\left(y_{1}, \ldots, y_{u}, x_{k}, \ldots, x_{l-1}\right) M: x_{l} \cap\left[\left(y_{1}, \ldots, y_{u}\right) M+\mathfrak{q}_{i}^{n_{i}} \cdots \mathfrak{q}_{j}^{n_{j}} M\right] \\
=\left(y_{1}, \ldots, y_{u}\right) M+\left(x_{k}, \ldots, x_{l-1}\right) \mathfrak{q}_{i}^{n_{i}} \cdots \mathfrak{q}_{k}^{n_{k}-1} \cdots \mathfrak{q}_{j}^{n_{j}} M
\end{gathered}
$$

for arbitrary integer $k \leq l \leq d$.

$\left(B_{i j}\right):$ If $y_{1}, \ldots, y_{u}$ is a subsystem of parameters for $M / \mathfrak{q}_{i} M$ and if $n_{k}>0$ for some integer $i \leq k \leq j$, then

$$
\begin{aligned}
{\left[\left(y_{1}, \ldots, y_{u-1}\right) M+\left(x_{k}, \ldots, x_{l}\right) \mathfrak{q}_{i}^{n_{i}} \cdots \mathfrak{q}_{j}^{n_{j}} M\right]: y_{u} } \\
=\left(x_{k}, \ldots, x_{l}\right)\left\{\left[\left(y_{1}, \ldots, y_{u-1}\right) M+\mathfrak{q}_{i}^{n_{i}} \cdots \mathfrak{q}_{j}^{n_{j}} M\right]: y_{u}\right\} \\
\quad+\left(y_{1}, \ldots, y_{u-1}\right) M: y_{u}
\end{aligned}
$$

for arbitrary integer $k \leq l \leq d$. In particular, by letting $l=d$, we have

$$
\begin{aligned}
{\left[\left(y_{1}, \ldots, y_{u-1}\right) M+\mathfrak{q}_{i}^{n_{i}} \cdots \mathfrak{q}_{k}^{n_{k}+1} \cdots \mathfrak{q}_{j}^{n_{j}} M\right]: y_{u} } \\
=\mathfrak{q}_{k}\left\{\left[\left(y_{1}, \ldots, y_{u-1}\right) M+\mathfrak{q}_{i}^{n_{i}} \cdots \mathfrak{q}_{j}^{n_{j}} M\right]: y_{u}\right\} \\
\quad+\left(y_{1}, \ldots, y_{u-1}\right) M: y_{u} .
\end{aligned}
$$


$\left(C_{i j}\right)$ : If $y_{1}, \ldots, y_{u}$ is a subsystem of parameters for $M / \mathfrak{q}_{i} M$ and if $n_{i}>0$, then

$$
\begin{aligned}
& {\left[\left(y_{1}, \ldots, y_{u-1}\right) M+\mathfrak{q}_{i}^{n_{i}} \cdots \mathfrak{q}_{j}^{n_{j}} M\right]: y_{u}} \\
& \quad \subseteq\left(y_{1}, \ldots, y_{u-1}\right) M: y_{u}+\mathfrak{q}_{i}^{n_{i}-1} \cdots \mathfrak{q}_{j}^{n_{j}} M .
\end{aligned}
$$

$\left(D_{i j}\right)$ : If $y_{1}, \ldots, y_{u}$ is a subsystem of parameters for $M / \mathfrak{q}_{i} M$ and if $n_{i}>0$, then

$$
\begin{aligned}
& {\left[\left(y_{1}, \ldots, y_{u-1}\right) M+\mathfrak{q}_{i}^{n_{i}} \cdots \mathfrak{q}_{j}^{n_{j}} M\right]: y_{u} \cap x_{i} M} \\
& \quad \subseteq x_{i}\left\{\left[\left(y_{1}, \ldots, y_{u-1}\right) M+\mathfrak{q}_{i}^{n_{i}-1} \cdots \mathfrak{q}_{j}^{n_{j}} M\right]: y_{u}\right\}+\left(y_{1}, \ldots, y_{u-1}\right) M
\end{aligned}
$$

$\left(E_{i j}\right)$ : Let $y_{1}, \ldots, y_{u}$ be a subsystem of parameters for $M / \mathfrak{q}_{k} M$ where $2 \leq k \leq i$ and $1 \leq u<k$. If $y_{u} \in \mathfrak{a}\left(M / \mathfrak{q}_{k} M\right)$ or $y_{u} \in \mathfrak{a}(M)$ and if $n_{i}>0$, then

$$
\begin{aligned}
& {\left[\left(y_{1}, \ldots, y_{v-1},\left\{x_{\lambda} \mid \lambda \in \Lambda\right\}\right) M+\mathfrak{q}_{i}^{n_{i}} \cdots \mathfrak{q}_{j}^{n_{j}} M\right]: y_{v} y_{u}} \\
& \quad=\left[\left(y_{1}, \ldots, y_{v-1},\left\{x_{\lambda} \mid \lambda \in \Lambda\right\}\right) M+\mathfrak{q}_{i}^{n_{i}} \cdots \mathfrak{q}_{j}^{n_{j}} M\right]: y_{u}
\end{aligned}
$$

for any $1 \leq v \leq u$ and $\Lambda \subseteq\{k, \ldots, i-1\}$.

Proof. We work by induction on $j-i$. First we assume that $i=j$.

$\left(A_{i i}\right)$ : Since $x_{i}, \ldots, x_{d}$ is a $d$-sequence on $M /\left(y_{1}, \ldots, y_{u}\right) M$, (3.6.1) coincides with Proposition 3.2

$\left(B_{i i}\right)$ : Let $a$ be an element in the left-hand side of (3.6.2) and put $y_{u} a=x_{l} b+c$ with $b \in \mathfrak{q}_{i}^{n_{i}} M$ and $c \in\left(y_{1}, \ldots, y_{u-1}\right) M+\left(x_{i}, \ldots, x_{l-1}\right) \mathfrak{q}_{i}^{n_{i}} M$. By using $\left(A_{i i}\right)$, we obtain

$$
\begin{aligned}
b & \in\left(y_{1}, \ldots, y_{u}, x_{i}, \ldots, x_{l-1}\right) M: x_{l} \cap \mathfrak{q}_{i}^{n_{i}} M \\
& \subseteq\left(y_{1}, \ldots, y_{u}\right) M+\left(x_{i}, \ldots, x_{l-1}\right) \mathfrak{q}_{i}^{n_{i}-1} M .
\end{aligned}
$$

Let $b=y_{u} a^{\prime}+c^{\prime}$ with $c^{\prime} \in\left(y_{1}, \ldots, y_{u-1}\right) M+\left(x_{i}, \ldots, x_{l-1}\right) \mathfrak{q}_{i}^{n_{i}-1} M$. Then $a^{\prime} \in$ $\left[\left(y_{1}, \ldots, y_{u-1}\right) M+\mathfrak{q}_{i}^{n_{i}} M\right]: y_{u}$ and

$$
a-x_{l} a^{\prime} \in\left[\left(y_{1}, \ldots, y_{u-1}\right) M+\left(x_{i}, \ldots, x_{l-1}\right) \mathfrak{q}_{i}^{n_{i}} M\right]: y_{u} .
$$

By induction on $l$, we find that $a$ is in the right-hand side of (3.6.2). The opposite inclusion is obvious.

$\left(C_{i i}\right)$ : By using $\left(B_{i i}\right)$ repeatedly, we have

$$
\begin{gathered}
{\left[\left(y_{1}, \ldots, y_{u-1}\right) M+\mathfrak{q}_{i}^{n_{i}} M\right]: y_{u}=\left(y_{1}, \ldots, y_{u-1}\right) M: y_{u}} \\
\quad+\mathfrak{q}_{i}^{n_{i}-1}\left\{\left[\left(y_{1}, \ldots, y_{u-1}\right) M+\mathfrak{q}_{i} M\right]: y_{u}\right\} \\
\subseteq\left(y_{1}, \ldots, y_{u-1}\right) M: y_{u}+\mathfrak{q}_{i}^{n_{i}-1} M .
\end{gathered}
$$

$\left(D_{i i}\right)$ : If $n_{i}=1$, then the right-hand side of (3.6.4) equals $\left(y_{1}, \ldots, y_{u-1}, x_{i}\right) M$ and hence contains the left-hand side.

Assume that $n_{i}>1$. Let $a$ be an element in $M$ such that $x_{i} a$ is in the left-hand side of (3.6.4). Then

$$
\begin{aligned}
y_{u} x_{i} a & \in\left[\left(y_{1}, \ldots, y_{u-1}\right) M+\mathfrak{q}_{i}^{n_{i}} M\right] \cap\left(y_{1}, \ldots, y_{u-1}, x_{i}\right) M \\
& =\left(y_{1}, \ldots, y_{u-1}\right) M+x_{i} \mathfrak{q}_{i}^{n_{i}-1} M
\end{aligned}
$$

because of $\left(A_{i i}\right)$. Hence

$$
\begin{aligned}
x_{i} a & \in\left[\left(y_{1}, \ldots, y_{u-1}\right) M+x_{i} \mathfrak{q}_{i}^{n_{i}-1} M\right]: y_{u} \\
& =\left(y_{1}, \ldots, y_{u-1}\right) M: y_{u}+x_{i}\left\{\left[\left(y_{1}, \ldots, y_{u-1}\right) M+\mathfrak{q}_{i}^{n_{i}-1} M\right]: y_{u}\right\} .
\end{aligned}
$$


Here we used $\left(B_{i i}\right)$. By applying Proposition 3.4 to a subsystem of parameters $y_{1}, \ldots, y_{u}, x_{i}$ for $M / \mathfrak{q}_{i+1} M$, we have

$$
\left(y_{1}, \ldots, y_{u-1}\right) M: y_{u} x_{i}=\left(y_{1}, \ldots, y_{u-1}\right) M: x_{i}
$$

and hence

$$
\begin{aligned}
\left(y_{1}, \ldots, y_{u-1}\right) M: y_{u} \cap x_{i} M & =x_{i}\left[\left(y_{1}, \ldots, y_{u-1}\right) M: y_{u} x_{i}\right] \\
& \subseteq\left(y_{1}, \ldots, y_{u-1}\right) M .
\end{aligned}
$$

Therefore

$$
\begin{aligned}
x_{i} a & \in x_{i}\left\{\left[\left(y_{1}, \ldots, y_{u-1}\right) M+\mathfrak{q}_{i}^{n_{i}-1} M\right]: y_{u}\right\}+\left(y_{1}, \ldots, y_{u-1}\right) M: y_{u} \cap x_{i} M \\
& \subseteq x_{i}\left\{\left[\left(y_{1}, \ldots, y_{u-1}\right) M+\mathfrak{q}_{i}^{n_{i}-1} M\right]: y_{u}\right\}+\left(y_{1}, \ldots, y_{u-1}\right) M .
\end{aligned}
$$

$\left(E_{i i}\right)$ : By using $\left(B_{i i}\right)$, we have

$$
\begin{aligned}
{\left[\left(y_{1}, \ldots, y_{v-1},\left\{x_{\lambda} \mid \lambda \in \Lambda\right\}\right) M+\mathfrak{q}_{i}^{n_{i}} M\right]: y_{v} y_{u} } \\
=\left(y_{1}, \ldots, y_{v-1},\left\{x_{\lambda} \mid \lambda \in \Lambda\right\}\right) M: y_{v} y_{u} \\
\quad+\mathfrak{q}_{i}^{n_{i}-1}\left\{\left[\left(y_{1}, \ldots, y_{v-1},\left\{x_{\lambda} \mid \lambda \in \Lambda\right\}\right) M+\mathfrak{q}_{i} M\right]: y_{v} y_{u}\right\} .
\end{aligned}
$$

Applying Proposition 3.4 to a subsystem of parameters $y_{1}, \ldots, y_{u}$ for $M / \mathfrak{q}_{k} M$ and two subsets of $\{k, \ldots, d\}: \Lambda$ and $\Lambda \cup\{i, \ldots, d\}$, we obtain

$$
\begin{aligned}
\left(y_{1}, \ldots,\right. & \left.y_{v-1},\left\{x_{\lambda} \mid \lambda \in \Lambda\right\}\right) M: y_{v} y_{u} \\
& +\mathfrak{q}_{i}^{n_{i}-1}\left\{\left[\left(y_{1}, \ldots, y_{v-1},\left\{x_{\lambda} \mid \lambda \in \Lambda\right\}\right) M+\mathfrak{q}_{i} M\right]: y_{v} y_{u}\right\} \\
= & \left(y_{1}, \ldots, y_{v-1},\left\{x_{\lambda} \mid \lambda \in \Lambda\right\}\right) M: y_{u} \\
& +\mathfrak{q}_{i}^{n_{i}-1}\left\{\left[\left(y_{1}, \ldots, y_{v-1},\left\{x_{\lambda} \mid \lambda \in \Lambda\right\}\right) M+\mathfrak{q}_{i} M\right]: y_{u}\right\} \\
= & {\left[\left(y_{1}, \ldots, y_{u-1},\left\{x_{\lambda} \mid \lambda \in \Lambda\right\}\right) M+\mathfrak{q}_{i}^{n_{i}} M\right]: y_{u} . }
\end{aligned}
$$

Thus (3.6.5) is shown.

Next we assume that $j>i$ and prove $\left(A_{i j}\right)-\left(E_{i j}\right)$. If $n_{i}=0$, then $\left(A_{i j}\right)$ and $\left(B_{i j}\right)$ are contained in $\left(A_{i+1, j}\right)$ and $\left(B_{i+1, j}\right)$, respectively. Therefore we may assume that $n_{i}>0$. Similarly we may also assume that $n_{j}>0$.

$\left(A_{i j}\right)$ : Let $a$ be an element in the left-hand side of (3.6.1). If $k=l=i$, then

$$
a \in\left(y_{1}, \ldots, y_{u}\right) M: x_{i} \cap\left(y_{1}, \ldots, y_{u}, x_{i}, \ldots, x_{d}\right) M=\left(y_{1}, \ldots, y_{u}\right) M .
$$

Otherwise, by using $\left(A_{i+1, j}\right)$, we have

$$
\begin{aligned}
a & \in\left(y_{1}, \ldots, y_{u}, x_{i}, x_{k}, \ldots, x_{l-1}\right) M: x_{l} \cap\left[\left(y_{1}, \ldots, y_{u}, x_{i}\right) M+\mathfrak{q}_{i+1}^{n_{i}+n_{i+1}} \cdots \mathfrak{q}_{j}^{n_{j}} M\right] \\
& = \begin{cases}\left(y_{1}, \ldots, y_{u}, x_{i}\right) M+\left(x_{i+1}, \ldots, x_{l-1}\right) \mathfrak{q}_{i+1}^{n_{i}+n_{i+1}-1} \cdots \mathfrak{q}_{j}^{n_{j}} M & \text { if } k \leq i+1, \\
\left(y_{1}, \ldots, y_{u}, x_{i}\right) M+\left(x_{k}, \ldots, x_{l-1}\right) \mathfrak{q}_{i+1}^{n_{i}+n_{i+1}} \cdots \mathfrak{q}_{k}^{n_{k}-1} \cdots \mathfrak{q}_{j}^{n_{j}} M & \text { if } k>i+1\end{cases} \\
& =\left(y_{1}, \ldots, y_{u}, x_{i}\right) M+\left(x_{k}, \ldots, x_{l-1}\right) \mathfrak{q}_{i}^{n_{i}} \cdots \mathfrak{q}_{k}^{n_{k}-1} \cdots \mathfrak{q}_{j}^{n_{j}} M .
\end{aligned}
$$

Taking the intersection with $\left(y_{1}, \ldots, y_{u}\right) M+\mathfrak{q}_{i}^{n_{i}} \cdots \mathfrak{q}_{j}^{n_{j}} M$, we obtain

$$
\begin{aligned}
a \in & \left(y_{1}, \ldots, y_{u}\right) M+\left(x_{k}, \ldots, x_{l-1}\right) \mathfrak{q}_{i}^{n_{i}} \cdots \mathfrak{q}_{k}^{n_{k}-1} \cdots \mathfrak{q}_{j}^{n_{j}} M \\
& +x_{i} M \cap\left[\left(y_{1}, \ldots, y_{u}\right) M+\mathfrak{q}_{i}^{n_{i}} \cdots \mathfrak{q}_{j}^{n_{j}} M\right] .
\end{aligned}
$$


Because of $\left(C_{i+1, j}\right)$,

$$
\begin{aligned}
x_{i} M \cap & {\left[\left(y_{1}, \ldots, y_{u}\right) M+\mathfrak{q}_{i}^{n_{i}} \cdots \mathfrak{q}_{j}^{n_{j}} M\right] } \\
= & x_{i} \mathfrak{q}_{i}^{n_{i}-1} \cdots \mathfrak{q}_{j}^{n_{j}} M \\
& +x_{i} M \cap\left[\left(y_{1}, \ldots, y_{u}\right) M+\mathfrak{q}_{i+1}^{n_{i}+n_{i+1}} \cdots \mathfrak{q}_{j}^{n_{j}} M\right] \\
= & x_{i} \mathfrak{q}_{i}^{n_{i}-1} \cdots \mathfrak{q}_{j}^{n_{j}} M+x_{i}\left\{\left[\left(y_{1}, \ldots, y_{u}\right) M+\mathfrak{q}_{i+1}^{n_{i}+n_{i+1}} \cdots \mathfrak{q}_{j}^{n_{j}} M\right]: x_{i}\right\} \\
\subseteq & x_{i} \mathfrak{q}_{i}^{n_{i}-1} \cdots \mathfrak{q}_{j}^{n_{j}} M+x_{i}\left[\left(y_{1}, \ldots, y_{u}\right) M: x_{i}+\mathfrak{q}_{i+1}^{n_{i}+n_{i+1}-1} \cdots \mathfrak{q}_{j}^{n_{j}} M\right] \\
\subseteq & \left(y_{1}, \ldots, y_{u}\right) M+x_{i} \mathfrak{q}_{i}^{n_{i}-1} \cdots \mathfrak{q}_{j}^{n_{j}} M .
\end{aligned}
$$

Therefore

$$
\begin{aligned}
a \in & \left(y_{1}, \ldots, y_{u}\right) M+\left(x_{k}, \ldots, x_{l-1}\right) \mathfrak{q}_{i}^{n_{i}} \cdots \mathfrak{q}_{k}^{n_{k}-1} \cdots \mathfrak{q}_{j}^{n_{j}} M \\
& +x_{i} \mathfrak{q}_{i}^{n_{i}-1} \cdots \mathfrak{q}_{j}^{n_{j}} M .
\end{aligned}
$$

If $k=i$, then the proof is completed. If $k>i$, then we work by induction on $n_{i}$. Let $a=x_{i} b+c$ with $b \in \mathfrak{q}_{i}^{n_{i}-1} \cdots \mathfrak{q}_{j}^{n_{j}} M$ and

$$
c \in\left(y_{1}, \ldots, y_{u}\right) M+\left(x_{k}, \ldots, x_{l-1}\right) \mathfrak{q}_{i}^{n_{i}} \cdots \mathfrak{q}_{k}^{n_{k}-1} \cdots \mathfrak{q}_{j}^{n_{j}} M
$$

If we apply Proposition 3.4 to a subsystem of parameters $y_{1}, \ldots, y_{u}, x_{k}, \ldots, x_{l-1}$, $x_{i}, x_{l}$ for $M / \mathfrak{q}_{l+1} M$, then we have

$$
b \in\left(y_{1}, \ldots, y_{u}, x_{k}, \ldots, x_{l-1}\right) M: x_{i} x_{l}=\left(y_{1}, \ldots, y_{u}, x_{k}, \ldots, x_{l-1}\right) M: x_{l} .
$$

If $n_{i}=1$, then $\left(A_{i+1, j}\right)$ says that

$$
\begin{aligned}
b & \in\left(y_{1}, \ldots, y_{u}, x_{k}, \ldots, x_{l-1}\right) M: x_{l} \cap \mathfrak{q}_{i+1}^{n_{i+1}} \cdots \mathfrak{q}_{j}^{n_{j}} M \\
& \subseteq\left(y_{1}, \ldots, y_{u}\right) M+\left(x_{k}, \ldots, x_{l-1}\right) \mathfrak{q}_{i+1}^{n_{i+1}} \cdots \mathfrak{q}_{k}^{n_{k}-1} \cdots \mathfrak{q}_{j}^{n_{j}} M
\end{aligned}
$$

and hence $a=x_{i} b+c$ is in the right-hand side of 3.6.1. If $n_{i}>1$, then we obtain

$$
\begin{aligned}
b & \in\left(y_{1}, \ldots, y_{u}, x_{k}, \ldots, x_{l-1}\right) M: x_{l} \cap \mathfrak{q}_{i}^{n_{i}-1} \cdots \mathfrak{q}_{j}^{n_{j}} M \\
& \subseteq\left(y_{1}, \ldots, y_{u}\right) M+\left(x_{k}, \ldots, x_{l-1}\right) \mathfrak{q}_{i}^{n_{i}-1} \cdots \mathfrak{q}_{k}^{n_{k}-1} \cdots \mathfrak{q}_{j}^{n_{j}} M
\end{aligned}
$$

by the induction hypothesis. Thus $a=x_{i} b+c$ is also in the right-hand side of (3.6.1).

$\left(B_{i j}\right)$ : Let $a$ be an element in the left-hand side of (3.6.2) and put $y_{u} a=x_{l} b+c$ with $b \in \mathfrak{q}_{i}^{n_{i}} \cdots \mathfrak{q}_{j}^{n_{j}} M$ and $c \in\left(y_{1}, \ldots, y_{u-1}\right) M+\left(x_{k}, \ldots, x_{l-1}\right) \mathfrak{q}_{i}^{n_{i}} \cdots \mathfrak{q}_{j}^{n_{j}} M$. Then

$$
\begin{aligned}
b & \in\left(y_{1}, \ldots, y_{u}, x_{k}, \ldots, x_{l-1}\right) M: x_{l} \cap \mathfrak{q}_{i}^{n_{i}} \cdots \mathfrak{q}_{j}^{n_{j}} M \\
& \subseteq\left(y_{1}, \ldots, y_{u}\right) M+\left(x_{k}, \ldots, x_{l-1}\right) \mathfrak{q}_{i}^{n_{i}} \cdots \mathfrak{q}_{k}^{n_{k}-1} \cdots \mathfrak{q}_{j}^{n_{j}} M .
\end{aligned}
$$

Here we used $\left(A_{i j}\right)$. If we put $b=y_{u} a^{\prime}+c^{\prime}$ with

$$
c^{\prime} \in\left(y_{1}, \ldots, y_{u-1}\right) M+\left(x_{k}, \ldots, x_{l-1}\right) \mathfrak{q}_{i}^{n_{i}} \cdots \mathfrak{q}_{k}^{n_{k}-1} \cdots \mathfrak{q}_{j}^{n_{j}} M
$$

then $a^{\prime} \in\left[\left(y_{1}, \ldots, y_{u-1}\right) M+\mathfrak{q}_{i}^{n_{i}} \cdots \mathfrak{q}_{j}^{n_{j}} M\right]: y_{u}$ and

$$
a-x_{l} a^{\prime} \in\left[\left(y_{1}, \ldots, y_{u-1}\right) M+\left(x_{k}, \ldots, x_{l-1}\right) \mathfrak{q}_{i}^{n_{i}} \cdots \mathfrak{q}_{j}^{n_{j}} M\right]: y_{u} .
$$

By induction on $l$, we find that $a$ is in the right-hand side of (3.6.2). The opposite inclusion is obvious.

$\left(C_{i j}\right)$ : We first show that

$$
\begin{gathered}
\left(y_{1}, \ldots, y_{u-1}, x_{i}\right) M: y_{u} \cap\left(y_{1}, \ldots, y_{u-1}, x_{i}, \ldots, x_{l}\right) M \\
\quad=\left(y_{1}, \ldots, y_{u-1}, x_{i}\right) M
\end{gathered}
$$


for all $i \leq l \leq d$. We work by induction on $l$. If $l=i$, then there exists nothing to prove. Assume that $l>i$ and let $a$ be an element in the left-hand side of (3.6.7). If we put $a=x_{l} b+c$ with $c \in\left(y_{1}, \ldots, y_{u-1}, x_{i}, \ldots, x_{l-1}\right) M$, then

$$
b \in\left(y_{1}, \ldots, y_{u-1}, x_{i}, \ldots, x_{l-1}\right) M: y_{u} x_{l}=\left(y_{1}, \ldots, y_{u-1}, x_{i}, \ldots, x_{l-1}\right) M: x_{l} .
$$

Here we applied Proposition 3.4 to a subsystem of parameters $y_{1}, \ldots, y_{u-1}, x_{i}$, $\ldots, x_{l-1}, y_{u}, x_{l}$ for $M / \mathfrak{q}_{l+1} M$. Thus we obtain

$$
\begin{aligned}
a=x_{l} b+c & \in\left(y_{1}, \ldots, y_{u-1}, x_{i}\right) M: y_{u} \cap\left(y_{1}, \ldots, y_{u-1}, x_{i}, \ldots, x_{l-1}\right) M \\
& =\left(y_{1}, \ldots, y_{u-1}, x_{i}\right) M
\end{aligned}
$$

by the induction hypothesis.

Next we show (3.6.3). By using $\left(B_{i j}\right)$, we may assume that $n_{i}=1$. Let $a$ be an element in the left-hand side of (3.6.3). Then

$$
\begin{aligned}
a & \in\left[\left(y_{1}, \ldots, y_{u-1}, x_{i}\right) M+\mathfrak{q}_{i+1}^{n_{i+1}+1} \cdots \mathfrak{q}_{j}^{n_{j}} M\right]: y_{u} \\
& \subseteq\left(y_{1}, \ldots, y_{u-1}, x_{i}\right) M: y_{u}+\mathfrak{q}_{i+1}^{n_{i+1}} \cdots \mathfrak{q}_{j}^{n_{j}} M
\end{aligned}
$$

because of $\left(C_{i+1, j}\right)$. On the other hand, since $n_{j}>0$, we obtain

$$
\begin{aligned}
a & \in\left[\left(y_{1}, \ldots, y_{u-1}\right) M+\mathfrak{q}_{i}^{2} M\right]: y_{u} \\
& \subseteq\left(y_{1}, \ldots, y_{u-1}\right) M: y_{u}+\mathfrak{q}_{i} M .
\end{aligned}
$$

Here we used $\left(C_{i i}\right)$. Hence

$$
\begin{aligned}
a & \in\left[\left(y_{1}, \ldots, y_{u-1}, x_{i}\right) M: y_{u}+\mathfrak{q}_{i+1}^{n_{i+1}} \cdots \mathfrak{q}_{j}^{n_{j}} M\right] \cap\left[\left(y_{1}, \ldots, y_{u-1}\right) M: y_{u}+\mathfrak{q}_{i} M\right] \\
& =\left(y_{1}, \ldots, y_{u-1}\right) M: y_{u}+\mathfrak{q}_{i+1}^{n_{i+1}} \cdots \mathfrak{q}_{j}^{n_{j}} M+\left(y_{1}, \ldots, y_{u-1}, x_{i}\right) M: y_{u} \cap \mathfrak{q}_{i} M \\
& =\left(y_{1}, \ldots, y_{u-1}\right) M: y_{u}+\mathfrak{q}_{i+1}^{n_{i+1}} \cdots \mathfrak{q}_{j}^{n_{j}} M+x_{i} M .
\end{aligned}
$$

Here we used (3.6.7). Taking the intersection with

$$
\left[\left(y_{1}, \ldots, y_{u-1}\right) M+\mathfrak{q}_{i+1}^{n_{i+1}} \cdots \mathfrak{q}_{j}^{n_{j}} M\right]: y_{u}
$$

we obtain

$$
\begin{aligned}
a \in & \left(y_{1}, \ldots, y_{u-1}\right) M: y_{u}+\mathfrak{q}_{i+1}^{n_{i+1}} \cdots \mathfrak{q}_{j}^{n_{j}} M \\
& +x_{i}\left\{\left[\left(y_{1}, \ldots, y_{u-1}\right) M+\mathfrak{q}_{i+1}^{n_{i+1}} \cdots \mathfrak{q}_{j}^{n_{j}} M\right]: y_{u} x_{i}\right\} .
\end{aligned}
$$

By applying $\left(E_{i+1, j}\right)$ to a subsystem of parameters $y_{1}, \ldots, y_{u}, x_{i}$ for $M / \mathfrak{q}_{i+1} M$, we have

$$
\left[\left(y_{1}, \ldots, y_{u-1}\right) M+\mathfrak{q}_{i+1}^{n_{i+1}} \cdots \mathfrak{q}_{j}^{n_{j}} M\right]: y_{u} x_{i}=\left[\left(y_{1}, \ldots, y_{u-1}\right) M+\mathfrak{q}_{i+1}^{n_{i+1}} \cdots \mathfrak{q}_{j}^{n_{j}} M\right]: x_{i} .
$$

Therefore $a \in\left(y_{1}, \ldots, y_{u-1}\right) M: y_{u}+\mathfrak{q}_{i+1}^{n_{i+1}} \cdots \mathfrak{q}_{j}^{n_{j}} M$.

$\left(D_{i j}\right)$ : Let $a$ be an element in $M$ such that $x_{i} a$ is in the left-hand side of (3.6.4). Then

$$
\begin{aligned}
y_{u} x_{i} a & \in x_{i} M \cap\left[\left(y_{1}, \ldots, y_{u-1}\right) M+\mathfrak{q}_{i}^{n_{i}} \cdots \mathfrak{q}_{j}^{n_{j}} M\right] \\
& \subseteq\left(y_{1}, \ldots, y_{u-1}\right) M+x_{i} \mathfrak{q}_{i}^{n_{i}-1} \cdots \mathfrak{q}_{j}^{n_{j}} M .
\end{aligned}
$$

Here we used $\left(A_{i j}\right)$. We put $y_{u} x_{i} a=x_{i} b+c$ with $b \in \mathfrak{q}_{i}^{n_{i}-1} \cdots \mathfrak{q}_{j}^{n_{j}} M$ and $c \in$ $\left(y_{1}, \ldots, y_{u-1}\right) M$. Then

$$
\begin{aligned}
b & \in\left(y_{1}, \ldots, y_{u}\right) M: x_{i} \cap \mathfrak{q}_{j} M \\
& \subseteq\left(y_{1}, \ldots, y_{u}\right) M: x_{i} \cap \mathfrak{q}_{i} M \\
& \subseteq\left(y_{1}, \ldots, y_{u}\right) M
\end{aligned}
$$


because $n_{j}>0$ and $x_{i}, \ldots, x_{d}$ is a $d$-sequence on $M /\left(y_{1}, \ldots, y_{u}\right) M$. If we put $b=y_{u} a^{\prime}+c^{\prime}$ with $c^{\prime} \in\left(y_{1}, \ldots, y_{u-1}\right) M$, then

$$
a^{\prime} \in\left[\left(y_{1}, \ldots, y_{u-1}\right) M+\mathfrak{q}_{i}^{n_{i}-1} \cdots \mathfrak{q}_{j}^{n_{j}} M\right]: y_{u}
$$

and

$$
\begin{aligned}
x_{i}\left(a-a^{\prime}\right) & \in\left(y_{1}, \ldots, y_{u-1}\right) M: y_{u} \cap x_{i} M \\
& \subseteq\left(y_{1}, \ldots, y_{u-1}\right) M .
\end{aligned}
$$

Here we used (3.6.6) again. Therefore

$$
x_{i} a \in\left(y_{1}, \ldots, y_{u-1}\right) M+x_{i}\left\{\left[\left(y_{1}, \ldots, y_{u-1}\right) M+\mathfrak{q}_{i}^{n_{i}-1} \cdots \mathfrak{q}_{j}^{n_{j}} M\right]: y_{u}\right\} .
$$

$\left(E_{i j}\right)$ : We may assume that $n_{i}=1$ in the same way as the proof of $\left(E_{i i}\right)$. We divide the proof into two cases.

First we assume that $n_{i+1}+\cdots+n_{j}=1$, that is, $n_{i+1}=\cdots=n_{j-1}=0$ and $n_{j}=1$. We show that

$$
\begin{aligned}
& {\left[\left(y_{1}, \ldots, y_{v-1},\left\{x_{\lambda} \mid \lambda \in \Lambda\right\}\right) M+\left(x_{i}, \ldots, x_{l-1}, x_{j}, \ldots, x_{d}\right) \mathfrak{q}_{i} M\right]: y_{v} y_{u}} \\
& \quad=\left[\left(y_{1}, \ldots, y_{v-1},\left\{x_{\lambda} \mid \lambda \in \Lambda\right\}\right) M+\left(x_{i}, \ldots, x_{l-1}, x_{j}, \ldots, x_{d}\right) \mathfrak{q}_{i} M\right]: y_{u}
\end{aligned}
$$

for all $i \leq l \leq j$ by descending induction on $l$. If $l=j$, then (3.6.8) coincides with $\left(E_{i i}\right)$. Assume that $l<j$ and let $a$ be an element in the left-hand side of (3.6.8). The induction hypothesis says that

$$
a \in\left[\left(y_{1}, \ldots, y_{v-1},\left\{x_{\lambda} \mid \lambda \in \Lambda\right\}\right) M+\left(x_{i}, \ldots, x_{l}, x_{j}, \ldots, x_{d}\right) \mathfrak{q}_{i} M\right]: y_{u} .
$$

We put $y_{u} a=x_{l} b+c$ with $b \in \mathfrak{q}_{i} M$ and

$$
c \in\left(y_{1}, \ldots, y_{v-1},\left\{x_{\lambda} \mid \lambda \in \Lambda\right\}\right) M+\left(x_{i}, \ldots, x_{l-1}, x_{j}, \ldots, x_{d}\right) \mathfrak{q}_{i} M .
$$

On the other hand, Proposition 3.4 says that

$$
\begin{aligned}
a & \in\left(y_{1}, \ldots, y_{v-1},\left\{x_{\lambda} \mid \lambda \in \Lambda\right\}, x_{i}, \ldots, x_{l-1}, x_{j}, \ldots, x_{d}\right) M: y_{v} y_{u} \\
& =\left(y_{1}, \ldots, y_{v-1},\left\{x_{\lambda} \mid \lambda \in \Lambda\right\}, x_{i}, \ldots, x_{l-1}, x_{j}, \ldots, x_{d}\right) M: y_{u} .
\end{aligned}
$$

Hence

$$
\begin{aligned}
b & \in\left(y_{1}, \ldots, y_{v-1},\left\{x_{\lambda} \mid \lambda \in \Lambda\right\}, x_{i}, \ldots, x_{l-1}, x_{j}, \ldots, x_{d}\right) M: x_{l} \cap \mathfrak{q}_{i} M \\
& \subseteq\left(y_{1}, \ldots, y_{v-1},\left\{x_{\lambda} \mid \lambda \in \Lambda\right\}, x_{i}, \ldots, x_{l-1}, x_{j}, \ldots, x_{d}\right) M
\end{aligned}
$$

because $x_{i}, \ldots, x_{j-1}$ is a $d$-sequence on

$$
M /\left(y_{1}, \ldots, y_{v-1},\left\{x_{\lambda} \mid \lambda \in \Lambda\right\}, x_{j}, \ldots, x_{d}\right) M .
$$

Therefore

$$
y_{u} a=x_{l} b+c \in\left(y_{1}, \ldots, y_{v-1},\left\{x_{\lambda} \mid \lambda \in \Lambda\right\}\right) M+\left(x_{i}, \ldots, x_{l-1}, x_{j}, \ldots, x_{d}\right) \mathfrak{q}_{i} M .
$$

Thus (3.6.8) is proved. If we put $l=i$, then we obtain

$$
\begin{aligned}
& {\left[\left(y_{1}, \ldots, y_{v-1},\left\{x_{\lambda} \mid \lambda \in \Lambda\right\}\right) M+\mathfrak{q}_{i} \mathfrak{q}_{j} M\right]: y_{v} y_{u}} \\
& \quad=\left[\left(y_{1}, \ldots, y_{v-1},\left\{x_{\lambda} \mid \lambda \in \Lambda\right\}\right) M+\mathfrak{q}_{i} \mathfrak{q}_{j} M\right]: y_{u} .
\end{aligned}
$$

Next we assume that $n_{i+1}+\cdots+n_{j}>1$. Let

$$
a \in\left[\left(y_{1}, \ldots, y_{v-1},\left\{x_{\lambda} \mid \lambda \in \Lambda\right\}\right) M+\mathfrak{q}_{i} \mathfrak{q}_{i+1}^{n_{i+1}} \cdots \mathfrak{q}_{j}^{n_{j}} M\right]: y_{v} y_{u} .
$$


Then $\left(E_{i+1, j}\right)$ says that

$$
\begin{aligned}
a & \in\left[\left(y_{1}, \ldots, y_{v-1},\left\{x_{\lambda} \mid \lambda \in \Lambda\right\}, x_{i}\right) M+\mathfrak{q}_{i+1}^{n_{i+1}+1} \cdots \mathfrak{q}_{j}^{n_{j}} M\right]: y_{v} y_{u} \\
& =\left[\left(y_{1}, \ldots, y_{v-1},\left\{x_{\lambda} \mid \lambda \in \Lambda\right\}, x_{i}\right) M+\mathfrak{q}_{i+1}^{n_{i+1}+1} \cdots \mathfrak{q}_{j}^{n_{j}} M\right]: y_{u} .
\end{aligned}
$$

Therefore

$$
\begin{aligned}
y_{u} a \in & {\left[\left(y_{1}, \ldots, y_{v-1},\left\{x_{\lambda} \mid \lambda \in \Lambda\right\}\right) M+\mathfrak{q}_{i} \mathfrak{q}_{i+1}^{n_{i+1}} \cdots \mathfrak{q}_{j}^{n_{j}} M\right]: y_{v} } \\
& \cap\left[\left(y_{1}, \ldots, y_{v-1},\left\{x_{\lambda} \mid \lambda \in \Lambda\right\}, x_{i}\right) M+\mathfrak{q}_{i+1}^{n_{i+1}+1} \cdots \mathfrak{q}_{j}^{n_{j}} M\right] \\
= & \left(y_{1}, \ldots, y_{v-1},\left\{x_{\lambda} \mid \lambda \in \Lambda\right\}\right) M+\mathfrak{q}_{i+1}^{n_{i+1}+1} \cdots \mathfrak{q}_{j}^{n_{j}} M \\
& +\left[\left(y_{1}, \ldots, y_{v-1},\left\{x_{\lambda} \mid \lambda \in \Lambda\right\}\right) M+\mathfrak{q}_{i} \mathfrak{q}_{i+1}^{n_{i+1}} \cdots \mathfrak{q}_{j}^{n_{j}} M\right]: y_{v} \cap x_{i} M \\
= & \left(y_{1}, \ldots, y_{v-1},\left\{x_{\lambda} \mid \lambda \in \Lambda\right\}\right) M+\mathfrak{q}_{i+1}^{n_{i+1}+1} \cdots \mathfrak{q}_{j}^{n_{j}} M \\
& +x_{i}\left\{\left[\left(y_{1}, \ldots, y_{v-1},\left\{x_{\lambda} \mid \lambda \in \Lambda\right\}\right) M+\mathfrak{q}_{i+1}^{n_{i+1}} \cdots \mathfrak{q}_{j}^{n_{j}} M\right]: y_{v}\right\} .
\end{aligned}
$$

Here we used $\left(D_{i j}\right)$ to show the second equality. We put $y_{u} a=x_{i} b+c$ with

$$
b \in\left[\left(y_{1}, \ldots, y_{v-1},\left\{x_{\lambda} \mid \lambda \in \Lambda\right\}\right) M+\mathfrak{q}_{i+1}^{n_{i+1}} \cdots \mathfrak{q}_{j}^{n_{j}} M\right]: y_{v}
$$

and

$$
c \in\left(y_{1}, \ldots, y_{v-1},\left\{x_{\lambda} \mid \lambda \in \Lambda\right\}\right) M+\mathfrak{q}_{i+1}^{n_{i+1}+1} \cdots \mathfrak{q}_{j}^{n_{j}} M .
$$

By applying $\left(C_{i+1, j}\right)$ to a subsystem of parameters $y_{1}, \ldots, y_{v-1}, y_{u},\left\{x_{\lambda} \mid \lambda \in\right.$ $\Lambda\}, x_{i}$ for $M / \mathfrak{q}_{i+1} M$, we obtain

$$
\begin{aligned}
b & \in\left[\left(y_{1}, \ldots, y_{v-1}, y_{u},\left\{x_{\lambda} \mid \lambda \in \Lambda\right\}\right) M+\mathfrak{q}_{i+1}^{n_{i+1}+1} \cdots \mathfrak{q}_{j}^{n_{j}} M\right]: x_{i} \\
& \subseteq\left(y_{1}, \ldots, y_{v-1}, y_{u},\left\{x_{\lambda} \mid \lambda \in \Lambda\right\}\right) M: x_{i}+\mathfrak{q}_{i+1}^{n_{i+1}} \cdots \mathfrak{q}_{j}^{n_{j}} M .
\end{aligned}
$$

On the other hand, since $n_{i+1}+\cdots+n_{j}>1$, we have

$$
\begin{gathered}
b \in\left[\left(y_{1}, \ldots, y_{v-1},\left\{x_{\lambda} \mid \lambda \in \Lambda\right\}\right) M+\mathfrak{q}_{i+1}^{2} M\right]: y_{v} \\
\subseteq\left(y_{1}, \ldots, y_{v-1},\left\{x_{\lambda} \mid \lambda \in \Lambda\right\}\right) M: y_{v}+\mathfrak{q}_{i+1} M
\end{gathered}
$$

by using $\left(C_{i+1, i+1}\right)$.

Furthermore, by applying Proposition 3.4 to a subsystem of parameters $y_{1}, \ldots$, $y_{v-1},\left\{x_{\lambda} \mid \lambda \in \Lambda\right\}, y_{v}, x_{i}$ for $M / \mathfrak{q}_{i+1} M$, we obtain

$$
\begin{aligned}
&\left(y_{1}, \ldots, y_{v-1},\left\{x_{\lambda} \mid \lambda \in \Lambda\right\}\right) M: y_{v} \\
& \subseteq\left(y_{1}, \ldots, y_{v-1},\left\{x_{\lambda} \mid \lambda \in \Lambda\right\}\right) M: y_{v} x_{i} \\
&=\left(y_{1}, \ldots, y_{v-1},\left\{x_{\lambda} \mid \lambda \in \Lambda\right\}\right) M: x_{i} .
\end{aligned}
$$

Hence, by taking the intersection of (3.6.10) and (3.6.11), we have

$$
\begin{aligned}
b \in & \left(y_{1}, \ldots, y_{v-1},\left\{x_{\lambda} \mid \lambda \in \Lambda\right\}\right) M: y_{v}+\mathfrak{q}_{i+1}^{n_{i+1}} \cdots \mathfrak{q}_{j}^{n_{j}} M \\
& +\left(y_{1}, \ldots, y_{v-1}, y_{u},\left\{x_{\lambda} \mid \lambda \in \Lambda\right\}\right) M: x_{i} \cap \mathfrak{q}_{i+1} M \\
& \subseteq \\
& \left(y_{1}, \ldots, y_{v-1},\left\{x_{\lambda} \mid \lambda \in \Lambda\right\}\right) M: y_{v}+y_{u} M+\mathfrak{q}_{i+1}^{n_{i+1}} \cdots \mathfrak{q}_{j}^{n_{j}} M .
\end{aligned}
$$

Here we apply Proposition 3.2 to a $d$-sequence $x_{i}, \ldots, x_{d}$ on

$$
M /\left(y_{1}, \ldots, y_{v-1}, y_{u},\left\{x_{\lambda} \mid \lambda \in \Lambda\right\}\right) M .
$$


Taking the intersection with (3.6.9), we obtain

$$
\begin{aligned}
b \in & \left(y_{1}, \ldots, y_{v-1},\left\{x_{\lambda} \mid \lambda \in \Lambda\right\}\right) M: y_{v}+\mathfrak{q}_{i+1}^{n_{i+1}} \cdots \mathfrak{q}_{j}^{n_{j}} M \\
& +\left[\left(y_{1}, \ldots, y_{v-1},\left\{x_{\lambda} \mid \lambda \in \Lambda\right\}\right) M+\mathfrak{q}_{i+1}^{n_{i+1}} \cdots \mathfrak{q}_{j}^{n_{j}} M\right]: y_{v} \cap y_{u} M \\
= & \left(y_{1}, \ldots, y_{v-1},\left\{x_{\lambda} \mid \lambda \in \Lambda\right\}\right) M: y_{v}+\mathfrak{q}_{i+1}^{n_{i+1}} \cdots \mathfrak{q}_{j}^{n_{j}} M \\
& +y_{u}\left\{\left[\left(y_{1}, \ldots, y_{v-1},\left\{x_{\lambda} \mid \lambda \in \Lambda\right\}\right) M+\mathfrak{q}_{i+1}^{n_{i+1}} \cdots \mathfrak{q}_{j}^{n_{j}} M\right]: y_{v} y_{u}\right\} \\
= & \left(y_{1}, \ldots, y_{v-1},\left\{x_{\lambda} \mid \lambda \in \Lambda\right\}\right) M: y_{v}+\mathfrak{q}_{i+1}^{n_{i+1}} \cdots \mathfrak{q}_{j}^{n_{j}} M .
\end{aligned}
$$

Here we used $\left(E_{i+1, j}\right)$ to show the last equality. By using (3.6.12) again, we find that

$$
y_{u} a=x_{i} b+c \in\left(y_{1}, \ldots, y_{v-1},\left\{x_{\lambda} \mid \lambda \in \Lambda\right\}\right) M+\mathfrak{q}_{i} \mathfrak{q}_{i+1}^{n_{i+1}} \cdots \mathfrak{q}_{j}^{n_{j}} M .
$$

That is,

$$
a \in\left[\left(y_{1}, \ldots, y_{v-1},\left\{x_{\lambda} \mid \lambda \in \Lambda\right\}\right) M+\mathfrak{q}_{i} \mathfrak{q}_{i+1}^{n_{i+1}} \cdots \mathfrak{q}_{j}^{n_{j}} M\right]: y_{u} .
$$

The opposite inclusion is obvious. The proof is completed.

Corollary 3.7. With the same notation as Theorem 3.6, we have

$$
\left[\left(y_{1}, \ldots, y_{u}\right) M+\mathfrak{q}_{i}^{n_{i}} \cdots \mathfrak{q}_{j}^{n_{j}} M\right]: x_{i-1}^{n_{i-1}}=\left[\left(y_{1}, \ldots, y_{u}\right) M+\mathfrak{q}_{i}^{n_{i}} \cdots \mathfrak{q}_{j}^{n_{j}} M\right]: \mathfrak{q}_{i-1}
$$

for any integers $2 \leq i \leq j \leq d, n_{i-1}>0, n_{i}, \ldots, n_{j} \geq 0$ and for any subsystem of parameters $y_{1}, \ldots, y_{u}$ for $M / \mathfrak{q}_{i-1} M$.

Proof. If $n_{i}=\cdots=n_{j}=0$, then the equality is trivial. Therefore we may assume that one of $n_{i}, \ldots, n_{j}$ is positive. We may also assume that $n_{i-1}=1$ by using Theorem 3.6 $\left(E_{i j}\right)$. Then we have

$$
\left[\left(y_{1}, \ldots, y_{u}\right) M+\mathfrak{q}_{i}^{n_{i}} \cdots \mathfrak{q}_{j}^{n_{j}} M\right]: x_{i-1} \subseteq\left(y_{1}, \ldots, y_{u}\right) M: x_{i-1}+\mathfrak{q}_{i}^{n_{i}-1} \cdots \mathfrak{q}_{j}^{n_{j}} M
$$

by applying Theorem $3.6\left(C_{i j}\right)$ to a subsystem of parameters $y_{1}, \ldots, y_{u}, x_{i-1}$ for $M / \mathfrak{q}_{i} M$. Since $x_{i-1}, \ldots, x_{d}$ is a $d$-sequence on $M /\left(y_{1}, \ldots, y_{u}\right) M$,

$$
\left(y_{1}, \ldots, y_{u}\right) M: x_{i-1} \subseteq\left(y_{1}, \ldots, y_{u}\right) M: \mathfrak{q}_{i-1} .
$$

Therefore

$$
\mathfrak{q}_{i-1}\left\{\left[\left(y_{1}, \ldots, y_{u}\right) M+\mathfrak{q}_{i}^{n_{i}} \cdots \mathfrak{q}_{j}^{n_{j}} M\right]: x_{i-1}\right\} \subseteq\left(y_{1}, \ldots, y_{u}\right) M+\mathfrak{q}_{i}^{n_{i}} \cdots \mathfrak{q}_{j}^{n_{j}} M .
$$

The opposite inclusion is trivial.

Corollary 3.8. With the same notation of Theorem 3.6, we let $k$ be an integer such that $1 \leq k \leq d$ and $y_{1}, \ldots, y_{u}$ a subsystem of parameters for $M / \mathfrak{q}_{k} M$. Assume that

$$
\left[\left(y_{1}, \ldots, y_{u-1}\right) M+\mathfrak{q}_{k} M\right]: y_{u}=\left(y_{1}, \ldots, y_{u-1}\right) M+\mathfrak{q}_{k} M
$$

Then

$$
\left(y_{1}, \ldots, y_{u-1},\left\{x_{\lambda} \mid \lambda \in \Lambda\right\}\right) M: y_{u}=\left(y_{1}, \ldots, y_{u-1},\left\{x_{\lambda} \mid \lambda \in \Lambda\right\}\right) M
$$

for any $\Lambda \subset\{k, \ldots, d\}$. Furthermore

$$
\begin{aligned}
& {\left[\left(y_{1}, \ldots, y_{u-1},\left\{x_{\lambda} \mid \lambda \in \Lambda\right\}\right) M+\mathfrak{q}_{i}^{n_{i}} \cdots \mathfrak{q}_{j}^{n_{j}} M\right]: y_{u}} \\
& \quad=\left(y_{1}, \ldots, y_{u-1},\left\{x_{\lambda} \mid \lambda \in \Lambda\right\}\right) M+\mathfrak{q}_{i}^{n_{i}} \cdots \mathfrak{q}_{j}^{n_{j}} M
\end{aligned}
$$

for any integers $k \leq i \leq j, n_{i}, \ldots, n_{j} \geq 0$, and $\Lambda \subseteq\{k, \ldots, i-1\}$. 
Proof. We first show (3.8.1) by descending induction on the number of elements in $\Lambda$. If $\Lambda=\{k, \ldots, d\}$, then there exists nothing to prove. Assume that $\Lambda \neq$ $\{k, \ldots, d\}$ and let $l$ be an element in $\{k, \ldots, d\} \backslash \Lambda$. Let $a$ be an element in the left-hand side of (3.8.1). Then

$$
a \in\left(y_{1}, \ldots, y_{u-1}, x_{l},\left\{x_{\lambda} \mid \lambda \in \Lambda\right\}\right) M: y_{u}=\left(y_{1}, \ldots, y_{u-1}, x_{l},\left\{x_{\lambda} \mid \lambda \in \Lambda\right\}\right) M
$$

because of the induction hypothesis. We put $a=x_{l} b+c$ with

$$
c \in\left(y_{1}, \ldots, y_{u-1},\left\{x_{\lambda} \mid \lambda \in \Lambda\right\}\right) M .
$$

Since $x_{l} \in \mathfrak{a}(M)$ or $x_{l} \in \mathfrak{a}\left(M / \mathfrak{q}_{l+1} M\right)$, we obtain

$$
b \in\left(y_{1}, \ldots, y_{u-1},\left\{x_{\lambda} \mid \lambda \in \Lambda\right\}\right) M: y_{u} x_{l}=\left(y_{1}, \ldots, y_{u-1},\left\{x_{\lambda} \mid \lambda \in \Lambda\right\}\right) M: x_{l}
$$

by using Proposition 3.4. Therefore $a=x_{l} b+c \in\left(y_{1}, \ldots, y_{u-1},\left\{x_{\lambda} \mid \lambda \in \Lambda\right\}\right) M$.

Next we show that (3.8.2). If $n_{i}=\cdots=n_{j}=0$, then the equality is trivial. We assume that $n_{i}, n_{j}>0$ and we work by induction on $j-i$. If $i=j$, then

$$
\begin{aligned}
{\left[\left(y_{1}, \ldots, y_{u-1},\left\{x_{\lambda} \mid \lambda \in \Lambda\right\}\right) M+\mathfrak{q}_{i}^{n_{i}} M\right]: y_{u} } \\
=\left(y_{1}, \ldots, y_{u-1},\left\{x_{\lambda} \mid \lambda \in \Lambda\right\}\right) M: y_{u} \\
\quad+\mathfrak{q}_{i}^{n_{i}-1}\left\{\left[\left(y_{1}, \ldots, y_{u-1},\left\{x_{\lambda} \mid \lambda \in \Lambda\right\}\right) M+\mathfrak{q}_{i} M\right]: y_{u}\right\} \\
=\left(y_{1}, \ldots, y_{u-1},\left\{x_{\lambda} \mid \lambda \in \Lambda\right\}\right) M+\mathfrak{q}_{i}^{n_{i}} M .
\end{aligned}
$$

Here we used Theorem [3.6 $\left(B_{i j}\right)$ and (3.8.1). Assume that $j>i$. We may assume that $n_{i}=1$ by using Theorem $3.6\left(B_{i j}\right)$. Let $a$ be an element of the left-hand side of (3.8.2). The induction hypothesis says that

$$
\begin{aligned}
& {\left[\left(y_{1}, \ldots, y_{u-1}, x_{i},\left\{x_{\lambda} \mid \lambda \in \Lambda\right\}\right) M+\mathfrak{q}_{i+1}^{n_{i+1}+1} \cdots \mathfrak{q}_{j}^{n_{j}} M\right]: y_{u}} \\
& \quad=\left(y_{1}, \ldots, y_{u-1}, x_{i},\left\{x_{\lambda} \mid \lambda \in \Lambda\right\}\right) M+\mathfrak{q}_{i+1}^{n_{i+1}+1} \cdots \mathfrak{q}_{j}^{n_{j}} M .
\end{aligned}
$$

Therefore

$$
\begin{aligned}
a \in & {\left[\left(y_{1}, \ldots, y_{u-1},\left\{x_{\lambda} \mid \lambda \in \Lambda\right\}\right) M+\mathfrak{q}_{i} \mathfrak{q}_{i+1}^{n_{i+1}} \cdots \mathfrak{q}_{j}^{n_{j}} M\right]: y_{u} } \\
& \cap\left[\left(y_{1}, \ldots, y_{u-1}, x_{i},\left\{x_{\lambda} \mid \lambda \in \Lambda\right\}\right) M+\mathfrak{q}_{i+1}^{n_{i+1}+1} \cdots \mathfrak{q}_{j}^{n_{j}} M\right] \\
= & \left(y_{1}, \ldots, y_{u-1},\left\{x_{\lambda} \mid \lambda \in \Lambda\right\}\right) M+\mathfrak{q}_{i+1}^{n_{i+1}+1} \cdots \mathfrak{q}_{j}^{n_{j}} M \\
& +\left[\left(y_{1}, \ldots, y_{u-1},\left\{x_{\lambda} \mid \lambda \in \Lambda\right\}\right) M+\mathfrak{q}_{i} \mathfrak{q}_{i+1}^{n_{i+1}} \cdots \mathfrak{q}_{j}^{n_{j}} M\right]: y_{u} \cap x_{i} M \\
\subseteq & \left(y_{1}, \ldots, y_{u-1},\left\{x_{\lambda} \mid \lambda \in \Lambda\right\}\right) M+\mathfrak{q}_{i+1}^{n_{i+1}+1} \cdots \mathfrak{q}_{j}^{n_{j}} M \\
& +x_{i}\left\{\left[\left(y_{1}, \ldots, y_{u-1},\left\{x_{\lambda} \mid \lambda \in \Lambda\right\}\right) M+\mathfrak{q}_{i+1}^{n_{i+1}} \cdots \mathfrak{q}_{j}^{n_{j}} M\right]: y_{u}\right\} \\
= & \left(y_{1}, \ldots, y_{u-1},\left\{x_{\lambda} \mid \lambda \in \Lambda\right\}\right) M+\mathfrak{q}_{i} \mathfrak{q}_{i+1}^{n_{i+1}} \cdots \mathfrak{q}_{j}^{n_{j}} M .
\end{aligned}
$$

Here we used Theorem $3.6\left(D_{i j}\right)$ and the induction hypothesis.

\section{The Proof of Theorem 1.1}

Before the proof of Theorem 1.1, we give some statements on $\mathbb{Z}^{r}$-graded rings. Let $R=\bigoplus_{n_{1}, \ldots, n_{r} \geq 0} R_{\left(n_{1}, \ldots, n_{r}\right)}$ be a Noetherian $\mathbb{Z}^{r}$-graded ring. For such a ring, let $R_{+}=\bigoplus_{\left(n_{1}, \ldots, n_{r}\right) \neq(0, \ldots, 0)} R_{\left(n_{1}, \ldots, n_{r}\right)}$.

Proposition 4.1. Let $M$ be a finitely generated graded $R$-module and $\mathfrak{b}$ an ideal in $R_{(0, \ldots, 0)}$. Then there exists an integer $n$ such that

$$
\left[H_{\mathfrak{b} R+R_{+}}^{p}(M)\right]_{\left(n_{1}, \ldots, n_{r}\right)}=0 \text { unless } n_{1}, \ldots, n_{r}<n
$$

for all $p \geq 0$. 
Proof. If $\mathfrak{b}=(0)$, then we can prove the assertion in the same way as $[28$, no. 66 Théorème 2]. The spectral sequence $E_{2}^{p q}=H_{\mathfrak{b} R}^{p} H_{R_{+}}^{q}(-) \Rightarrow H_{\mathfrak{b} R+R_{+}}^{p+q}(-)$ says that the assertion holds in general.

Let $\varphi: \mathbb{Z}^{r} \rightarrow \mathbb{Z}^{s}$ be a group homomorphism satisfying $\varphi\left(\mathbb{N}^{r}\right) \subseteq \mathbb{N}^{s}$. We put

$$
R^{\varphi}=\bigoplus_{m_{1}, \ldots, m_{s} \geq 0}\left(\bigoplus_{\varphi\left(n_{1}, \ldots, n_{r}\right)=\left(m_{1}, \ldots, m_{s}\right)} R_{\left(n_{1}, \ldots, n_{r}\right)}\right),
$$

which is a $\mathbb{Z}^{s}$-graded ring. For a graded $R$-module $M$, let

$$
M^{\varphi}=\bigoplus_{m_{1}, \ldots, m_{s} \in \mathbb{Z}}\left(\bigoplus_{\varphi\left(n_{1}, \ldots, n_{r}\right)=\left(m_{1}, \ldots, m_{s}\right)} M_{\left(n_{1}, \ldots, n_{r}\right)}\right),
$$

which is a graded $R^{\varphi}$-module. We know that

$$
\left[H_{\mathfrak{b} R+R_{+}}^{p}(M)\right]^{\varphi}=H_{\mathfrak{b} R^{\varphi}+\left(R^{\varphi}\right)_{+}}^{p}\left(M^{\varphi}\right)
$$

for any ideal $\mathfrak{b}$ in $R_{(0, \ldots, 0)}$. See Lemma 1.1 of [15].

The following proposition is contained in the proof of [15, Theorem 2.2].

Proposition 4.2. Let $M=\bigoplus_{n_{1}, \ldots, n_{r} \geq 0} M_{\left(n_{1}, \ldots, n_{r}\right)}$ be a finitely generated graded $R$-module and $\mathfrak{b}$ an ideal in $R_{(0, \ldots, 0)}$. We put

$$
S=\bigoplus_{n_{1}, \ldots, n_{r+1} \geq 0} R_{\left(n_{1}, \ldots, n_{r-1}, n_{r}+n_{r+1}\right)}
$$

and

$$
N=\bigoplus_{n_{1}, \ldots, n_{r+1} \geq 0} M_{\left(n_{1}, \ldots, n_{r-1}, n_{r}+n_{r+1}\right)} .
$$

Then $S$ is a Noetherian $\mathbb{Z}^{r+1}$-graded ring and $N$ a finitely generated graded $S$ module.

If there exists an integer $p_{0}$ such that

$$
H_{\mathfrak{b} R+R_{+}}^{p}(M)=0 \quad \text { for all } p>p_{0},
$$

then

$$
H_{\mathfrak{b} S+S_{+}}^{p}(N)=0 \quad \text { for all } p>p_{0}+1
$$

$$
\left[H_{\mathfrak{b} R+R_{+}}^{p}(M)\right]_{\left(n_{1}, \ldots, n_{r}\right)}=0 \text { unless } n_{1}, \ldots, n_{r}<0
$$

for all $p$, then

$$
\left[H_{\mathfrak{b} S+S_{+}}^{p}(N)\right]_{\left(n_{1}, \ldots, n_{r+1}\right)}=0 \quad \text { unless } n_{1}, \ldots, n_{r+1}<0
$$

for all $p$. If, in addition, there exist integers $p_{0}>0$ and $n_{0}<0$ such that

$$
\left[H_{\mathfrak{b} R+R_{+}}^{p}(M)\right]_{\left(n_{1}, \ldots, n_{r}\right)}=0 \quad \text { whenever } n_{1}+\cdots+n_{r} \leq n_{0}
$$

for all $p<p_{0}$, then

$$
\left[H_{\mathfrak{b} S+S_{+}}^{p}(N)\right]_{\left(n_{1}, \ldots, n_{r+1}\right)}=0 \quad \text { whenever } n_{1}+\cdots+n_{r+1} \leq n_{0}
$$

for all $p<p_{0}+1$. 
Proof. It is easy to show that $S$ is a $\mathbb{Z}^{r+1}$-graded ring and $N$ a graded $S$-module. First we show that $S$ is Noetherian. To do this, we may assume that $r=1$ without loss of generality. Since $R$ is Noetherian, $R_{0}$ is also and $R$ is generated by finitely generated $R_{0}$-modules $R_{1}, \ldots, R_{k}$ over $R_{0}$. Then $S=S_{(0,0)}\left[S_{\left(n_{1}, n_{2}\right)} \mid n_{1}+n_{2} \leq k\right]$. Indeed, if $i+j>k$, then $R_{i+j}=R_{1} R_{i+j-1}+\cdots+R_{k} R_{i+j-k}$. Therefore

$$
S_{(i, j)}= \begin{cases}\sum_{l=1}^{k} S_{(l, 0)} S_{(i-l, j)}, & \text { if } i \geq k \\ \sum_{l=1}^{i} S_{(l, 0)} S_{(i-l, j)}+\sum_{m=1}^{k-i} S_{(i, m)} S_{(0, j-m)}, & \text { if } i<k .\end{cases}
$$

We can show that $S_{(i, j)} \subset S_{(0,0)}\left[S_{\left(n_{1}, n_{2}\right)} \mid n_{1}+n_{2} \leq k\right]$ by induction on $i+j$. Similarly we can prove that $N$ is a finitely generated $S$-module.

Next we consider local cohomology modules. Let

$$
I=\bigoplus_{n_{1}, \ldots, n_{r} \geq 0, n_{r+1}>0} R_{\left(n_{1}, \ldots, n_{r-1}, n_{r}+n_{r+1}\right)}
$$

and

$$
L_{1}=\bigoplus_{n_{1}, \ldots, n_{r} \geq 0, n_{r+1}>0} M_{\left(n_{1}, \ldots, n_{r-1}, n_{r}+n_{r+1}\right)} .
$$

If we put $\varphi\left(n_{1}, \ldots, n_{r}\right)=\left(n_{1}, \ldots, n_{r}, 0\right)$, then $S / I \cong R^{\varphi}$ and $N / L_{1} \cong M^{\varphi}$. Therefore

$$
\left[H_{\mathfrak{b} S+S_{+}}^{p}\left(N / L_{1}\right)\right]_{\left(n_{1}, \ldots, n_{r+1}\right)}= \begin{cases}{\left[H_{\mathfrak{b} R+R_{+}}^{p}(M)\right]_{\left(n_{1}, \ldots, n_{r}\right)},} & \text { if } n_{r+1}=0 \\ 0, & \text { otherwise }\end{cases}
$$

for all $p$. Similarly we put

$$
L_{2}=\bigoplus_{n_{1}, \ldots, n_{r-1}, n_{r+1} \geq 0, n_{r}>0} M_{\left(n_{1}, \ldots, n_{r-1}, n_{r}+n_{r+1}\right)} .
$$

Then

$$
\left[H_{\mathfrak{b} S+S_{+}}^{p}\left(N / L_{2}\right)\right]_{\left(n_{1}, \ldots, n_{r+1}\right)}= \begin{cases}{\left[H_{\mathfrak{b} R+R_{+}}^{p}(M)\right]_{\left(n_{1}, \ldots, n_{r-1}, n_{r+1}\right)},} & \text { if } n_{r}=0 \\ 0, & \text { otherwise }\end{cases}
$$

for all $p$.

There exist two long exact sequences of local cohomology modules

$$
\cdots \rightarrow H_{\mathfrak{b} S+S_{+}}^{p-1}\left(N / L_{i}\right) \rightarrow H_{\mathfrak{b} S+S_{+}}^{p}\left(L_{i}\right) \rightarrow H_{\mathfrak{b} S+S_{+}}^{p}(N) \rightarrow H_{\mathfrak{b} S+S_{+}}^{p}\left(N / L_{i}\right) \rightarrow \cdots
$$

for $i=1$ and 2 . On the other hand, $L_{1} \cong L_{2}(0, \ldots, 0,1,-1)$.

Assume that (4.2.1) holds. If $p>p_{0}+1$, then

$$
\begin{aligned}
{\left[H_{\mathfrak{b} S+S_{+}}^{p}(N)\right]_{\left(n_{1}, \ldots, n_{r+1}\right)} } & \cong\left[H_{\mathfrak{b} S+S_{+}}^{p}\left(L_{1}\right)\right]_{\left(n_{1}, \ldots, n_{r+1}\right)} \\
& \cong\left[H_{\mathfrak{b} S+S_{+}}^{p}\left(L_{2}\right)\right]_{\left(n_{1}, \ldots, n_{r-1}, n_{r}+1, n_{r+1}-1\right)} \\
& \cong\left[H_{\mathfrak{b} S+S_{+}}^{p}(N)\right]_{\left(n_{1}, \ldots, n_{r-1}, n_{r}+1, n_{r+1}-1\right)} \\
& \cong \cdots=0
\end{aligned}
$$

Here we used Proposition 4.1. 
Next we assume that (4.2.2) holds for all $p$. Unless $n_{1}, \ldots, n_{r}<0$, then

$$
\begin{aligned}
& {\left[_{\mathfrak{b} S+S_{+}}^{p}(N)\right]_{\left(n_{1}, \ldots, n_{r+1}\right)} \cong\left[H_{\mathfrak{b} S+S_{+}}^{p}\left(L_{1}\right)\right]_{\left(n_{1}, \ldots, n_{r+1}\right)} } \\
& \cong\left[H_{\mathfrak{b} S+S_{+}}^{p}\left(L_{2}\right)\right]_{\left(n_{1}, \ldots, n_{r-1}, n_{r}+1, n_{r+1}-1\right)} \\
& \cong\left[H_{\mathfrak{b} S+S_{+}}^{p}(N)\right]_{\left(n_{1}, \ldots, n_{r-1}, n_{r}+1, n_{r+1}-1\right)} \\
& \cong \cdots=0 .
\end{aligned}
$$

We can also show that $\left[H_{\mathfrak{b} S+S_{+}}^{p}(L)\right]_{\left(n_{1}, \ldots, n_{r+1}\right)}=0$ if $n_{r+1} \geq 0$. In addition, we also assume that (4.2.3) holds for all $p<p_{0}$. If $p<p_{0}+1, n_{1}+\cdots+n_{r+1} \leq n_{0}$, and $n_{1}, \ldots, n_{r+1}<0$, then

$$
\begin{aligned}
& {\left[_{\mathfrak{b} S+S_{+}}^{p}(N)\right]_{\left(n_{1}, \ldots, n_{r+1}\right)} \cong\left[H_{\mathfrak{b} S+S_{+}}^{p}\left(L_{1}\right)\right]_{\left(n_{1}, \ldots, n_{r+1}\right)} } \\
& \cong\left[H_{\mathfrak{b} S+S_{+}}^{p}\left(L_{2}\right)\right]_{\left(n_{1}, \ldots, n_{r-1}, n_{r}+1, n_{r+1}-1\right)} \\
& \subseteq\left[H_{\mathfrak{b} S+S_{+}}^{p}(N)\right]_{\left(n_{1}, \ldots, n_{r-1}, n_{r}+1, n_{r+1}-1\right)} \\
& \cong \cdots=0 .
\end{aligned}
$$

The proof is completed.

Let $\mathfrak{b}_{1}, \ldots, \mathfrak{b}_{r}$ be ideals in $A$. The multigraded Rees algebra of $A$ (for short, the multi-Rees algebra) with respect to them is defined to be

$$
R\left(\mathfrak{b}_{1}, \ldots, \mathfrak{b}_{r}\right)=A\left[\mathfrak{b}_{1} T_{1}, \ldots, \mathfrak{b}_{r} T_{r}\right],
$$

where $T_{1}, \ldots, T_{r}$ are indeterminates. If $\mathfrak{b}_{1}, \ldots, \mathfrak{b}_{r}$ are of positive height, then $\operatorname{dim} R\left(\mathfrak{b}_{1}, \ldots, \mathfrak{b}_{r}\right)=\operatorname{dim} A+r$. See Proposition 1.17 of [15]. For an $A$-module $M$, let $R_{M}\left(\mathfrak{b}_{1}, \ldots, \mathfrak{b}_{r}\right)$ denote the $R\left(\mathfrak{b}_{1}, \ldots, \mathfrak{b}_{r}\right)$-module

$$
\bigoplus_{n_{1}, \ldots, n_{r} \geq 0} \mathfrak{b}_{1}^{n_{1}} \cdots \mathfrak{b}_{r}^{n_{r}} M T_{1}^{n_{1}} \cdots T_{r}^{n_{r}}
$$

Recently Hyry gives the following theorem.

Theorem 4.3 ([16, Corollary 2.10]). Let $\mathfrak{b}_{1}, \ldots, \mathfrak{b}_{r}$ be ideals in $A$ of positive height. If the multi-Rees algebra $R\left(\mathfrak{b}_{1}, \ldots, \mathfrak{b}_{r}\right)$ is Cohen-Macaulay, then the ordinary Rees algebra $R\left(\mathfrak{b}_{1} \cdots \mathfrak{b}_{r}\right)$ is also Cohen-Macaulay.

We start to prove Theorem [1.1.

Theorem 4.4. Let $M$ be a finitely generated $A$-module and $x_{t}, \ldots, x_{d}$ elements in $A$. We fix integers $t \leq s+1<d, \alpha_{t}, \ldots, \alpha_{s}>0$, and $\alpha_{s+1} \geq d-s-1$. Let $\mathfrak{q}_{i}=\left(x_{i}, \ldots, x_{d}\right)$ for all $t \leq i \leq s+1$. We put

$$
S=A\left[\mathfrak{q}_{t} T_{t, 1}, \ldots, \mathfrak{q}_{t} T_{t, \alpha_{t}}, \mathfrak{q}_{t+1} T_{t+1,1}, \ldots, \mathfrak{q}_{s} T_{s, \alpha_{s}}, \mathfrak{q}_{s+1} T_{s+1,1}, \ldots, \mathfrak{q}_{s+1} T_{s+1, \alpha_{s+1}}\right]
$$

and $N$ the $S$-module $R_{M}\left(\mathfrak{q}_{t}, \ldots, \mathfrak{q}_{s+1}\right)$. If the sequence $x_{t}, \ldots, x_{d}$ satisfies the following six conditions:

(1) the sequence $x_{i}, \ldots, x_{d}$ is a d-sequence on $M /\left(x_{\lambda}^{n_{\lambda}} \mid \lambda \in \Lambda\right) M$ for all $t \leq i \leq$ $s+1, n_{t}, \ldots, n_{i-1}>0$, and $\Lambda \subseteq\{t, \ldots, i-1\}$

(2) the sequence $x_{i}, \ldots, x_{d-1}$ is a $d$-sequence on $M /\left(\left\{x_{\lambda} \mid \lambda \in \Lambda\right\}, x_{d}\right) M$ for all $t \leq i \leq s+1, n_{t}, \ldots, n_{i-1}>0$, and $\Lambda \subseteq\{t, \ldots, i-1\}$

(3) the sequence $x_{s+1}, \ldots, x_{d}$ is a u.s.d-sequence on $M /\left(x_{\lambda}^{n_{\lambda}} \mid \lambda \in \Lambda\right) M$ for all $n_{t}, \ldots, n_{s}>0$ and $\Lambda \subseteq\{t, \ldots, s\}$; 
(4) the equality

$$
\begin{gathered}
\left(\left\{x_{\lambda}^{n_{\lambda}} \mid \lambda \in \Lambda\right\}, x_{k}, \ldots, x_{l-1}\right) M: x_{l} \cap\left[\left(x_{\lambda}^{n_{\lambda}} \mid \lambda \in \Lambda\right) M+\mathfrak{q}_{i}^{n_{i}} \cdots \mathfrak{q}_{s+1}^{n_{s+1}} M\right] \\
=\left(x_{\lambda}^{n_{\lambda}} \mid \lambda \in \Lambda\right) M+\left(x_{k}, \ldots, x_{l-1}\right) \mathfrak{q}_{i}^{n_{i}} \cdots \mathfrak{q}_{k}^{n_{k}-1} \cdots \mathfrak{q}_{s+1}^{n_{s+1}} M
\end{gathered}
$$

holds for any integers $t \leq i \leq k \leq s+1, k \leq l \leq d, n_{t}, \ldots, n_{i-1}, n_{k}>0$, $n_{i}, \ldots, n_{k-1}, n_{k+1}, \ldots, n_{s+1} \geq 0$, and $\Lambda \subseteq\{t, \ldots, i-1\}$;

(5) the equality

$$
\begin{aligned}
& {\left[\left(x_{\lambda}^{n_{\lambda}} \mid \lambda \in \Lambda\right) M+\mathfrak{q}_{i}^{n_{i}} \cdots \mathfrak{q}_{s+1}^{n_{s+1}} M\right]: x_{i-1}^{n_{i-1}}} \\
& \quad=\left[\left(x_{\lambda}^{n_{\lambda}} \mid \lambda \in \Lambda\right) M+\mathfrak{q}_{i}^{n_{i}} \cdots \mathfrak{q}_{s+1}^{n_{s+1}} M\right]: \mathfrak{q}_{i-1}
\end{aligned}
$$

holds for any $t<i \leq s+1, n_{t}, \ldots, n_{i-1}>0, n_{i}, \ldots, n_{s+1} \geq 0$, and $\Lambda \subset\{t, \ldots, i-2\}$

(6) $0:_{M} x_{d} \subseteq 0:_{M} x_{t}$,

then

$$
\begin{aligned}
& H_{\mathfrak{q}_{t} S+S_{+}}^{0}(N)=0 \underset{M}{:} x_{d}, \\
& H_{\mathfrak{q}_{t} S+S_{+}}^{p}(N)=0 \quad \text { for } p \neq 0, d-t+1+\alpha_{t}+\cdots+\alpha_{s+1},
\end{aligned}
$$

and

$$
\left[H_{\mathfrak{q}_{t} S+S_{+}}^{d-t+1+\alpha_{t}+\cdots+\alpha_{s+1}}(N)\right]_{\left(n_{t, 1}, \ldots, n_{s+1, \alpha_{s+1}}\right)}=0,
$$

unless $n_{t, 1}, \ldots, n_{s+1, \alpha_{s+1}}<0$.

Proof. We show that 4.4.1 -4.4.3 by descending induction on $t$. First we note that $d-s \geq 2$ because of the assumption. Furthermore $0:_{M} x_{t} \subset \cdots \subset 0:_{M} x_{d}$ because $x_{t}, \ldots, x_{d}$ is a $d$-sequence on $M$. Therefore (1) and (6) say that $0:_{M} x_{t}=$ $\cdots=0:_{M} x_{d}$. Without loss of generality, we may assume that $0:_{M} x_{d}=0$. Indeed, assumptions (1)-(6) hold on $\bar{M}=M / 0:_{M} x_{d}$. For example,

$$
\begin{gathered}
{\left[\left(\left\{x_{\lambda}^{n_{\lambda}} \mid \lambda \in \Lambda\right\}, x_{k}, \ldots, x_{l-1}\right) M+0 \underset{M}{:} x_{t}\right]: x_{l}} \\
\quad=\left(\left\{x_{\lambda}^{n_{\lambda}} \mid \lambda \in \Lambda\right\}, x_{k}, \ldots, x_{l-1}\right) M: x_{l}^{2} \\
\quad=\left(\left\{x_{\lambda}^{n_{\lambda}} \mid \lambda \in \Lambda\right\}, x_{k}, \ldots, x_{l-1}\right) M: x_{l}
\end{gathered}
$$

because $0:_{M} x_{t} \subset 0:_{M} x_{l}$. Hence

$$
\begin{aligned}
\left(\left\{x_{\lambda}^{n_{\lambda}} \mid \lambda\right.\right. & \left.\left.\in \Lambda\}, x_{k}, \ldots, x_{l-1}\right) M\right]: x_{l} \cap\left[\left(x_{\lambda}^{n_{\lambda}} \mid \lambda \in \Lambda\right) M+\mathfrak{q}_{i}^{n_{i}} \cdots \mathfrak{q}_{s+1}^{n_{s+1}} M+0 \underset{M}{:} x_{t}\right] \\
= & \left(\left\{x_{\lambda}^{n_{\lambda}} \mid \lambda \in \Lambda\right\}, x_{k}, \ldots, x_{l-1}\right) M: x_{l} \cap\left[\left(x_{\lambda}^{n_{\lambda}} \mid \lambda \in \Lambda\right) M+\mathfrak{q}_{i}^{n_{i}} \cdots \mathfrak{q}_{s+1}^{n_{s+1}} M\right] \\
& +0 \dot{M} x_{t} \\
= & \left(x_{\lambda}^{n_{\lambda}} \mid \lambda \in \Lambda\right) M+\left(x_{k}, \ldots, x_{l-1}\right) \mathfrak{q}_{i}^{n_{i}} \cdots \mathfrak{q}_{k}^{n_{k}-1} \cdots \mathfrak{q}_{s+1}^{n_{s+1}} M+0 \underset{M}{:} x_{t} .
\end{aligned}
$$

Thus (4) holds on $\bar{M}$. Similarly we can show that (1)-(3) and (5) hold on $\bar{M}$. Of course $0: \bar{M} x_{t}=0: \bar{M} x_{d}=0$. On the other hand, if $\bar{N}$ denotes the $S$-module $R_{\bar{M}}\left(\mathfrak{q}_{t}, \ldots, \mathfrak{q}_{s+1}\right)$, then there exists an exact sequence of $S$-modules

$$
0 \rightarrow 0 \underset{\dot{M}}{:} x_{t} \rightarrow N \rightarrow \bar{N} \rightarrow 0 .
$$

Since $0:_{M} x_{t}$ is annihilated by $\mathfrak{q}_{t} S+S_{+}$,

$$
0 \rightarrow 0 \underset{\dot{M}}{:} x_{t} \rightarrow H_{\mathfrak{q}_{t} S+S_{+}}^{0}(N) \rightarrow H_{\mathfrak{q}_{t} S+S_{+}}^{0}(\bar{N}) \rightarrow 0
$$


is exact and

$$
H_{\mathfrak{q}_{t} S+S_{+}}^{p}(\bar{N}) \cong H_{\mathfrak{q}_{t} S+S_{+}}^{p}(N) \text { for all } p>0 .
$$

Thus if the assertion holds for $\bar{M}$, then the one holds for $M$.

From now on we assume that $0:_{M} x_{t}=\cdots=0:_{M} x_{d}=0$. Because of Proposition 4.2, we may assume that $\alpha_{t}=\cdots=\alpha_{s}=1$ and $\alpha_{s+1}=d-s-1$. For the simplicity, we write $T_{t}=T_{t, 1}, \ldots, T_{s+1}=T_{s+1,1}, T_{s+2}=T_{s+1,2}, \ldots$, $T_{d-1}=T_{s+1, d-s-1}$.

Assume that $t=s+1$ and put $R=A\left[\mathfrak{q}_{s+1} T_{s+1}\right]$. Then we know that

$$
\left[H_{\mathfrak{q}_{s+1} R+R_{+}}^{p}\left(R_{M}\left(\mathfrak{q}_{s+1}\right)\right)\right]_{n}=0 \quad \text { unless } 2-p \leq n \leq-1
$$

for all $p<d-s+1$,

$$
\left[H_{\mathfrak{q}_{s+1} R+R_{+}}^{d-s+1}\left(R_{M}\left(\mathfrak{q}_{t+1}\right)\right)\right]_{n}=0 \quad \text { unless } n<0
$$

and

$$
H_{\mathfrak{q}_{s+1} R+R_{+}}^{p}\left(R_{M}\left(\mathfrak{q}_{t+1}\right)\right)=0 \text { for all } p>d-s+1 .
$$

See [12, Theorem 4.1]. By using Proposition 4.2, repeatedly, we find that

$$
H_{\mathfrak{q}_{s+1} S+S_{+}}^{p}(N)=0 \text { for } p \neq 2 d-2 s-1
$$

and

$$
\left[H_{\mathfrak{q}_{s+1} S+S_{+}}^{2 d-2 s-1}(N)\right]_{\left(n_{s+1}, \ldots, n_{d-1}\right)}=0 \quad \text { unless } n_{s+1}, \ldots, n_{d-1}<0 .
$$

Thus we obtain 4.4.1)- 4.4.3).

Next we assume that $t<s+1$. Then $x_{t}^{m} M: x_{t+1}=x_{t}^{m} M: x_{d}$ for any $m>0$. Indeed, if $a \in x_{t}^{m} M: x_{d}$ and we put $x_{d} a=x_{t}^{m} b$, then $b \in x_{d} M: x_{t}^{m} \subseteq x_{d} M: x_{t+1}$ because of (2). Let $x_{t+1} b=x_{d} c$. Then $x_{t+1} x_{d} a=x_{t}^{m} x_{t+1} b=x_{t}^{m} x_{d} c$. Therefore $x_{t+1} a-x_{t}^{m} c \in 0: x_{M} x_{d}=0$ and hence $a \in x_{t}^{m} M: x_{t+1}$. Thus the sequence $x_{t+1}$, $\ldots, x_{d}$ satisfies (1)-(6) on $M$ and on $M / x_{t}^{m} M$ for any $m>0$.

Let $R=A\left[\mathfrak{q}_{t+1} T_{t+1}, \ldots, \mathfrak{q}_{s+1} T_{s+1}, \ldots, \mathfrak{q}_{s+1} T_{d-1}\right]$ and

$$
Y=\bigoplus_{n_{t+1}, \ldots, n_{d-1} \geq 0}\left[\mathfrak{q}_{t+1}^{n_{t+1}} \cdots \mathfrak{q}_{s+1}^{n_{s+1}+\cdots+n_{d-1}} M: \mathfrak{q}_{t}\right] T_{t+1}^{n_{t+1}} \cdots T_{d-1}^{n_{d-1}}
$$

Then assumption (5) gives an exact sequence of $R$-modules

$$
0 \rightarrow Y \stackrel{x_{t}^{m}}{\longrightarrow} R_{M}\left(\mathfrak{q}_{t+1} \cdots \mathfrak{q}_{s+1}\right) \rightarrow R_{M / x_{t}^{m} M}\left(\mathfrak{q}_{t+1}, \ldots, \mathfrak{q}_{s+1}\right) \rightarrow 0
$$

and hence $Y$ is finitely generated over $R$. The induction hypothesis says that

$$
\begin{aligned}
& H_{\mathfrak{q}_{t+1} R+R_{+}}^{p}\left(R_{M}\left(\mathfrak{q}_{t+1}, \ldots, \mathfrak{q}_{s+1}\right)\right)=0 \text { for } p \neq 2 d-2 t-1 \\
& {\left[H_{\mathfrak{q}_{t+1} R+R_{+}}^{2 d-2 t-1}\left(R_{M}\left(\mathfrak{q}_{t+1}, \ldots, \mathfrak{q}_{s+1}\right)\right)\right]_{\left(n_{t+1}, \ldots, n_{d-1}\right)}=0}
\end{aligned}
$$

unless $n_{t+1}, \ldots, n_{d-1}<0$,

$$
H_{\mathfrak{q}_{t+1} R+R_{+}}^{p}\left(R_{M / x_{t}^{m} M}\left(\mathfrak{q}_{t+1}, \ldots, \mathfrak{q}_{s+1}\right)\right)=0 \text { for } p \neq 0,2 d-2 t-1,
$$

and

$$
\left[H_{\mathfrak{q}_{t+1} R+R_{+}}^{2 d-2 t-1}\left(R_{M / x_{t}^{m} M}\left(\mathfrak{q}_{t+1}, \ldots, \mathfrak{q}_{s+1}\right)\right)\right]_{\left(n_{t+1}, \ldots, n_{d-1}\right)}=0
$$

unless $n_{t+1}, \ldots, n_{d-1}<0$. The spectral sequence

$$
E_{2}^{p q}=H_{x_{t}}^{p} H_{\mathfrak{q}_{t+1} R+R_{+}}^{q}(-) \Rightarrow H_{\mathfrak{q}_{t} R+R_{+}}^{p+q}(-)
$$


gives a short exact sequence

$$
0 \rightarrow H_{x_{t}}^{1} H_{\mathfrak{q}_{t+1} R+R_{+}}^{p-1}(-) \rightarrow H_{\mathfrak{q}_{t} R+R_{+}}^{p}(-) \rightarrow H_{x_{t}}^{0} H_{\mathfrak{q}_{t+1} R+R_{+}}^{p}(-) \rightarrow 0 .
$$

By using it, we obtain

$$
\begin{aligned}
& H_{\mathfrak{q}_{t} R+R_{+}}^{p}\left(R_{M}\left(\mathfrak{q}_{t+1}, \ldots, \mathfrak{q}_{s+1}\right)\right)=0 \text { for } p \neq 2 d-2 t-1,2 d-2 t \\
& {\left[H_{\mathfrak{q}_{t} R+R_{+}}^{2 d-2 t}\left(R_{M}\left(\mathfrak{q}_{t+1}, \ldots, \mathfrak{q}_{s+1}\right)\right)\right]_{\left(n_{t+1}, \ldots, n_{d-1}\right)}=0}
\end{aligned}
$$

unless $n_{t+1}, \ldots, n_{d-1}<0$,

$$
H_{\mathfrak{q}_{t} R+R_{+}}^{p}\left(R_{M / x_{t}^{m} M}\left(\mathfrak{q}_{t+1}, \ldots, \mathfrak{q}_{s+1}\right)\right)=0 \quad \text { for } p \neq 0,2 d-2 t-1,
$$

and

$$
\left[H_{\mathfrak{q}_{t} R+R_{+}}^{2 d-2 t-1}\left(R_{M / x_{t}^{m} M}\left(\mathfrak{q}_{t+1}, \ldots, \mathfrak{q}_{s+1}\right)\right)\right]_{\left(n_{t+1}, \ldots, n_{d-1}\right)}=0
$$

unless $n_{t+1}, \ldots, n_{d-1}<0$. Therefore

$$
\begin{aligned}
& H_{\mathfrak{q}_{t} R+R_{+}}^{p}(Y)=0 \text { for } p \neq 1,2 d-2 t-1,2 d-2 t, \\
& {\left[H_{\mathfrak{q}_{t} R+R_{+}}^{2 d-2 t}(Y)\right]_{\left(n_{t+1}, \ldots, n_{d-1}\right)}=0 \quad \text { unless } n_{t+1}, \ldots, n_{d-1}<0,}
\end{aligned}
$$

and

$$
0 \rightarrow H_{\mathfrak{q}_{t} R+R_{+}}^{2 d-2 t-1}(Y) \rightarrow H_{\mathfrak{q}_{t} R+R_{+}}^{2 d-2 t-1}\left(R_{M}\left(\mathfrak{q}_{t+1}, \ldots, \mathfrak{q}_{s+1}\right)\right)
$$

is exact. We show that $H_{\mathfrak{q}_{t} R+R_{+}}^{2 d-2 t-1}(Y)=0$. Let $E=H_{\mathfrak{q}_{t} R+R_{+}}^{2 d-2 t-1}\left(R_{M}\left(\mathfrak{q}_{t+1}, \ldots, \mathfrak{q}_{s+1}\right)\right)$. Because of (5),

$$
\mathfrak{q}_{t} Y \subseteq R_{M}\left(\mathfrak{q}_{t+1}, \ldots, \mathfrak{q}_{s+1}\right) \subseteq Y
$$

Therefore

$$
H_{\mathfrak{q}_{t} R+R_{+}}^{p}\left(Y / R_{M}\left(\mathfrak{q}_{t+1}, \ldots, \mathfrak{q}_{s+1}\right)\right) \cong H_{R_{+}}^{p}\left(Y / R_{M}\left(\mathfrak{q}_{t+1}, \ldots, \mathfrak{q}_{s+1}\right)\right)
$$

Let

$$
\begin{aligned}
& f_{2 t+2}=x_{t+1} T_{t+1}, \\
& f_{2 t+3}=x_{t+2} T_{t+1} \text {, } \\
& f_{2 t+4}=x_{t+3} T_{t+1}+x_{t+2} T_{t+2}, \\
& \text { : } \\
& f_{d+t+1}=x_{d} T_{t+1}+x_{d-1} T_{t+2}+\cdots, \\
& f_{d+t+2}=x_{d} T_{t+1}+\cdots, \\
& \begin{array}{r}
\vdots \\
f_{2 d-2}=
\end{array} \\
& x_{d} T_{d-2}+x_{d-1} T_{d-1}, \\
& f_{2 d-1}= \\
& x_{d} T_{d-1} \text {. }
\end{aligned}
$$

Then $\sqrt{R_{+}}=\sqrt{\left(f_{2 t+2}, \ldots, f_{2 d-1}\right) R}$. The proof is quite similar to [11, Lemma 3.2]. We omit it. Therefore

$$
H_{\mathfrak{q}_{t} R+R_{+}}^{p}\left(Y / R_{M}\left(\mathfrak{q}_{t+1}, \ldots, \mathfrak{q}_{s+1}\right)\right)=0 \quad \text { for } p>2 d-2 t-2
$$

and hence

$$
H_{\mathfrak{q}_{t} R+R_{+}}^{2 d-2 t-2}\left(Y / R_{M}\left(\mathfrak{q}_{t+1}, \ldots, \mathfrak{q}_{s+1}\right)\right) \rightarrow E \rightarrow H_{\mathfrak{q}_{t} R+R_{+}}^{2 d-2 t-1}(Y) \rightarrow 0
$$

is exact. Thus

$$
H_{\mathfrak{q}_{t} R+R_{+}}^{2 d-2 t-1}\left(Y / R_{M}\left(\mathfrak{q}_{t+1}, \ldots, \mathfrak{q}_{s+1}\right)\right) \rightarrow E \stackrel{x_{t}^{m}}{\longrightarrow} E
$$


is exact. Since the first term of (4.4.4) is annihilated by $x_{t}$, we obtain $0:_{E} x_{t}^{m}=$ $0:_{E} x_{t}$. Therefore $x_{t} E=0$ and hence $H_{\mathfrak{q}_{t} R+R_{+}}^{2 d-2 t-1}(Y)=0$ because $E=\bigcup_{m>0} 0:_{E} x_{t}^{m}$. Since $R=S / \mathfrak{q}_{t} T_{t} S, Y$ is also an $S$-module and

$$
\begin{aligned}
& H_{\mathfrak{q}_{t} S+S_{+}}^{p}(Y)=0 \text { for } p \neq 1,2 d-2 t, \\
& {\left[H_{\mathfrak{q}_{t} S+S_{+}}^{2 d-2 t}(Y)\right]_{\left(n_{t}, \ldots, n_{d-1}\right)}=0 \text { unless } n_{t}=0, n_{t-1}, \ldots, n_{d-1}<0 .}
\end{aligned}
$$

Let $S^{\prime}=A\left[\mathfrak{q}_{t+1} T_{t}, \mathfrak{q}_{t+1} T_{t+1}, \ldots, \mathfrak{q}_{s} T_{s}, \mathfrak{q}_{s+1} T_{s+1}, \ldots, \mathfrak{q}_{s+1} T_{d-1}\right]$. Then the induction hypothesis says that

$$
H_{\mathfrak{q}_{t+1} S^{\prime}+S_{+}^{\prime}}^{p}\left(R_{M / x_{t} M}\left(\mathfrak{q}_{t+1}, \mathfrak{q}_{t+1}, \ldots, \mathfrak{q}_{s+1}\right)\right)=0 \quad \text { for } p \neq 0,2 d-2 t
$$

and

$$
\left[H_{\mathfrak{q}_{t+1} S^{\prime}+S_{+}^{\prime}}^{2 d-2 t}\left(R_{M / x_{t} M}\left(\mathfrak{q}_{t+1}, \mathfrak{q}_{t+1}, \ldots, \mathfrak{q}_{s+1}\right)\right)\right]_{\left(n_{t}, \ldots, n_{d-1}\right)}=0
$$

unless $n_{t}, \ldots, n_{d-1}<0$. Since $S^{\prime}$ is an $A$-subalgebra of $S$, we can regard the $S$ module $R_{M / x_{t} M}\left(\mathfrak{q}_{t}, \ldots, \mathfrak{q}_{t+1}\right)$ as an $S^{\prime}$-module and there exists an $S^{\prime}$-isomorphism

$$
R_{M / x_{t} M}\left(\mathfrak{q}_{t}, \mathfrak{q}_{t+1}, \ldots, \mathfrak{q}_{s+1}\right) \cong R_{M / x_{t} M}\left(\mathfrak{q}_{t+1}, \mathfrak{q}_{t+1}, \ldots, \mathfrak{q}_{s+1}\right) .
$$

Since $\left(x_{t}, x_{t} T_{t}\right) R_{M / x_{t} M}\left(\mathfrak{q}_{t}, \ldots, \mathfrak{q}_{s+1}\right)=0$,

$$
\begin{aligned}
& H_{\mathfrak{q}_{t} S+S_{+}}^{p}\left(R_{M / x_{t} M}\left(\mathfrak{q}_{t}, \ldots, \mathfrak{q}_{s+1}\right)\right) \\
& \quad=H_{\left(\mathfrak{q}_{t+1} S^{\prime}+S_{+}^{\prime}\right) S}^{p}\left(R_{M / x_{t} M}\left(\mathfrak{q}_{t}, \ldots, \mathfrak{q}_{s+1}\right)\right) \\
& \quad=H_{\mathfrak{q}_{t+1} S^{\prime}+S_{+}^{\prime}}^{p}\left(R_{M / x_{t} M}\left(\mathfrak{q}_{t}, \ldots, \mathfrak{q}_{s+1}\right)\right)=0
\end{aligned}
$$

for $p \neq 0,2 d-2 t$ and

$$
\left[H_{\mathfrak{q}_{t} S+S_{+}}^{2 d-2 t}\left(R_{M / x_{t} M}\left(\mathfrak{q}_{t}, \ldots, \mathfrak{q}_{s+1}\right)\right)\right]_{\left(n_{t}, \ldots, n_{d-1}\right)}=0 \quad \text { unless } n_{t}, \ldots, n_{d-1}<0 .
$$

Let $X$ be the kernel of the natural epimorphism $N \rightarrow R_{M / x_{t} M}\left(\mathfrak{q}_{t}, \ldots, \mathfrak{q}_{s+1}\right)$. Then there exists an exact sequence of $S$-modules

$$
0 \rightarrow X \rightarrow N \rightarrow R_{M / x_{t} M}\left(\mathfrak{q}_{t}, \ldots, \mathfrak{q}_{s+1}\right) \rightarrow 0 .
$$

Since

$$
x_{t} M \cap \mathfrak{q}_{t}^{n_{t}} \cdots \mathfrak{q}_{s+1}^{n_{s+1}+\cdots+n_{d-1}} M=x_{t} \mathfrak{q}_{t}^{n_{t}-1} \cdots \mathfrak{q}_{s+1}^{n_{s+1}+\cdots+n_{d-1}} M
$$

if $n_{t}>0$,

$$
\bigoplus_{n_{t}>0} X_{\left(n_{t}, \ldots, n_{d-1}\right)}=x_{t} T_{t} N
$$

and there exists an exact sequence

$$
0 \rightarrow N(-1,0, \ldots, 0) \stackrel{x_{t} T_{t}}{\longrightarrow} X \stackrel{x_{t}^{-1}}{\longrightarrow} Y \rightarrow 0 .
$$

Because of (4.4.5) and (4.4.6),

$$
0 \rightarrow H_{\mathfrak{q}_{t} S+S_{+}}^{p}(N)(-1,0, \ldots, 0) \stackrel{x_{t} T_{t}}{\longrightarrow} H_{\mathfrak{q}_{t} S+S_{+}}^{p}(N)
$$

is exact if $3 \leq p<2 d-2 t+1$ or $p>2 d-2 t+1$. Since $H_{\mathfrak{q}_{t} S+S_{+}}^{p}(N)$ is annihilated by some power of $x_{t} T_{t}$ elementwise,

$$
H_{\mathfrak{q}_{t} S+S_{+}}^{p}(N)=0 \quad \text { if } 3 \leq p<2 d-2 t+1 \text { or } p>2 d-2 t+1 .
$$

Furthermore

$$
H_{\mathfrak{q}_{t} S+S_{+}}^{2 d-2 t}(Y) \rightarrow H_{\mathfrak{q}_{t} S+S_{+}}^{2 d-2 t+1}(N)(-1,0, \ldots, 0) \rightarrow H_{\mathfrak{q}_{t} S+S_{+}}^{2 d-22+1}(X) \rightarrow 0
$$


and

$$
H_{\mathfrak{q}_{t} S+S_{+}}^{2 d-2 t}\left(R_{M / x_{t} M}\left(\mathfrak{q}_{t}, \ldots, \mathfrak{q}_{s+1}\right)\right) \rightarrow H_{\mathfrak{q}_{t} S+S_{+}}^{2 d-2 t+1}(X) \rightarrow H_{\mathfrak{q}_{t} S+S_{+}}^{2 d-2 t+1}(N) \rightarrow 0
$$

are exact. Unless $n_{t}, \ldots, n_{d-1}<0$, then we obtain

$$
\begin{aligned}
{\left[H_{\mathfrak{q}_{t} S+S_{+}}^{2 d-2 t+1}(N)\right]_{\left(n_{t}, \ldots, n_{d-1}\right)} } & \cong\left[H_{\mathfrak{q}_{t} S+S_{+}}^{2 d-2 t+1}(X)\right]_{\left(n_{t}+1, n_{t+1}, \ldots, n_{d-1}\right)} \\
& \cong\left[H_{\mathfrak{q}_{t} S+S_{+}}^{2 d-2 t+1}(N)\right]_{\left(n_{t}+1, n_{t+1}, \ldots, n_{d-1}\right)} \\
& \cong \cdots=0 .
\end{aligned}
$$

Thus (4.4.2) is proved.

Finally we show that $x_{s} T_{s}, x_{s+1} T_{s+1}, x_{s+2}$ is a regular sequence on $N$. Since $x_{s}$ is regular on $M, x_{s} T_{s}$ is regular on $N$.

Let $a T_{t}^{n_{t}} \cdots T_{d-1}^{n_{d-1}} \in x_{s} T_{s} N: x_{s+1} T_{s+1}$. If $n_{s}=0$, then $x_{s+1} a=0$ and hence $a=0$. If $n_{s}>0$, then

$$
a \in x_{s} M: x_{s+1} \cap \mathfrak{q}_{t}^{n_{t}} \cdots \mathfrak{q}_{s+1}^{n_{s+1}+\cdots+n_{d-1}} M=x_{s} \mathfrak{q}_{t}^{n_{t}} \cdots \mathfrak{q}_{s}^{n_{s}-1} \mathfrak{q}_{s+1}^{n_{s+1}+\cdots+n_{d-1}} M .
$$

Here we used (4). Hence $a T_{t}^{n_{t}} \cdots T_{d-1}^{n_{d-1}} \in x_{s} T_{s} N$.

Let $a T_{t}^{n_{t}} \cdots T_{d-1}^{n_{d-1}} \in\left(x_{s} T_{s}, x_{s+1} T_{s+1}\right) N: x_{s+2}$. If $n_{s}=n_{s+1}=0$, then $x_{s+2} a=0$ and hence $a=0$. If $n_{s}>0$ and $n_{s+1}=0$, then $a \in x_{s} M: x_{s+2}$. Because of (3), we have $x_{s} M: x_{s+1}=x_{s} M: x_{s+2}$. Hence

$$
a \in x_{s} M: x_{s+1} \cap \mathfrak{q}_{t}^{n_{t}} \cdots \mathfrak{q}_{s+1}^{n_{s+1}+\cdots+n_{d-1}} M=x_{s} \mathfrak{q}_{t}^{n_{t}} \cdots \mathfrak{q}_{s}^{n_{s}-1} \mathfrak{q}_{s+1}^{n_{s+1}+\cdots+n_{d-1}} M,
$$

that is, $a T_{t}^{n_{t}} \cdots T_{d-1}^{n_{d-1}} \in x_{s} T_{s} N$. If $n_{s}=0$ and $n_{s+1}>0$, then

$$
a \in x_{s+1} M: x_{s+2} \cap \mathfrak{q}_{t}^{n_{t}} \cdots \mathfrak{q}_{s+1}^{n_{s+1}+\cdots+n_{d-1}} M=x_{s+1} \mathfrak{q}_{t}^{n_{t}} \cdots \mathfrak{q}_{s+1}^{n_{s+1}+\cdots+n_{d-1}-1} M
$$

and hence $a T_{t}^{n_{t}} \cdots T_{d-1}^{n_{d-1}} \in x_{s+1} T_{s+1} N$. If $n_{s}, n_{s+1}>0$, then

$$
\begin{aligned}
a & \in\left(x_{s}, x_{s+1}\right) M: x_{s+2} \cap \mathfrak{q}_{t}^{n_{t}} \cdots \mathfrak{q}_{s+1}^{n_{s+1}+\cdots+n_{d-1}} M \\
& =\left(x_{s}, x_{s+1}\right) \mathfrak{q}_{t}^{n_{t}} \cdots \mathfrak{q}_{s}^{n_{s}-1} \mathfrak{q}_{s+1}^{n_{s+1}+\cdots+n_{d-1}} M \\
& =x_{s} \mathfrak{q}_{t}^{n_{t}} \cdots \mathfrak{q}_{s}^{n_{s}-1} \mathfrak{q}_{s+1}^{n_{s+1}+\cdots+n_{d-1}} M+x_{s+1} \mathfrak{q}_{t}^{n_{t}} \cdots \mathfrak{q}_{s+1}^{n_{s+1}+\cdots+n_{d-1}-1} M .
\end{aligned}
$$

Therefore $a T_{t}^{n_{t}} \cdots T_{d-1}^{n_{d-1}} \in\left(x_{s} T_{s}, x_{s+1} T_{s+1}\right) N$.

Thus we obtain

$$
H_{\mathfrak{q}_{t} S+S_{+}}^{p}(N)=0 \quad \text { for } p<3 .
$$

The proof is completed.

Corollary 4.5. Let $A$ be a Noetherian local ring of dimension $d \geq 2$ and $x_{1}, \ldots$, $x_{d}$ a p-standard system of parameters of type $s$ for $A$. We put $\mathfrak{q}_{i}=\left(x_{i}, \ldots, x_{d}\right)$ for all $1 \leq i \leq s+1$. If $s<d-1$ and $(0): x_{d}=0$, then the Rees algebra $R\left(\mathfrak{q}_{1} \cdots \mathfrak{q}_{s} \mathfrak{q}_{s+1}^{d-s-1}\right)$ is a Cohen-Macaulay ring. If, in addition, A/ $\mathfrak{q}_{t}$ is CohenMacaulay for some $1<t \leq s+1$, then $R\left(\mathfrak{q}_{t} \cdots \mathfrak{q}_{s} \mathfrak{q}_{s+1}^{d-s-1}\right)$ is a Cohen-Macaulay ring.

Proof. In this case Propositions 3.3, 3.5, Theorem 3.6, and Corollary 3.7 say that $x_{1}, \ldots, x_{d}$ satisfies assumptions (1)-(5) of Theorem 4.4. Moreover $(0): x_{1} \supseteq$ $(0): x_{d}=0$. Thus we find that $A\left[\mathfrak{q}_{1} T_{1}, \ldots, \mathfrak{q}_{s} T_{s}, \mathfrak{q}_{s+1} T_{s+1}, \ldots, \mathfrak{q}_{s+1} T_{d-1}\right]$ is CohenMacaulay by using Theorem 4.4 Hyry's theorem says that $R\left(\mathfrak{q}_{1} \cdots \mathfrak{q}_{s} \mathfrak{q}_{s+1}^{d-s-1}\right)$ is Cohen-Macaulay. 
Assume that $A / \mathfrak{q}_{t}$ is Cohen-Macaulay. That is, $x_{1}, \ldots, x_{t-1}$ is a regular sequence on $A / \mathfrak{q}_{t}$. We show that

$$
\left(x_{1}, \ldots, x_{i}\right): x_{d}=\left(x_{1}, \ldots, x_{i}\right) \text { for } 1 \leq i \leq t-1
$$

by induction on $i$. If $i=0$, then there exists nothing to prove. Assume that $i>0$ and let $a \in\left(x_{1}, \ldots, x_{i}\right): x_{d}$. If we put $x_{d} a=b+x_{i} c$ with $b \in\left(x_{1}, \ldots, x_{i-1}\right)$, then

$$
\begin{aligned}
c & \in\left(x_{1}, \ldots, x_{i-1}, x_{d}\right): x_{i} \\
& =\left(x_{1}, \ldots, x_{i-1}, x_{d}\right) .
\end{aligned}
$$

Here we used Corollary 3.8. Let $c=b^{\prime}+x_{d} a^{\prime}$ with $b^{\prime} \in\left(x_{1}, \ldots, x_{i-1}\right)$. Then

$$
a-x_{i} a^{\prime} \in\left(x_{1}, \ldots, x_{i-1}\right): x_{d}=\left(x_{1}, \ldots, x_{i-1}\right)
$$

because of the induction hypothesis. Therefore $a \in\left(x_{1}, \ldots, x_{i}\right)$. Thus $x_{t}, \ldots, x_{d}$ satisfies the assumptions of Theorem 4.4 on $\bar{A}=A /\left(x_{1}, \ldots, x_{t-1}\right)$. Therefore

$$
\bar{A}\left[\mathfrak{q}_{t} \bar{A} T_{t}, \ldots, \mathfrak{q}_{s} \bar{A} T_{s}, \mathfrak{q}_{s+1} \bar{A} T_{s+1}, \ldots, \mathfrak{q}_{s+1} \bar{A} T_{d-1}\right]
$$

is a Cohen-Macaulay ring and hence $R\left(\mathfrak{q}_{t} \cdots \mathfrak{q}_{s} \mathfrak{q}_{s+1}^{d-s-1} \bar{A}\right)$ is also. Corollary 3.8 also says that $x_{1}, \ldots, x_{t-1}$ is a regular sequence on $A$ and on $A /\left(\mathfrak{q}_{t} \cdots \mathfrak{q}_{s} \mathfrak{q}_{s+1}^{d-s-1}\right)^{n}$ for all $n>0$. Taking Koszul cohomology of a short exact sequence

$$
0 \rightarrow R\left(\mathfrak{q}_{t} \cdots \mathfrak{q}_{s} \mathfrak{q}_{s+1}^{d-s-1}\right) \rightarrow A[T] \rightarrow \bigoplus_{n>0}\left(A /\left(\mathfrak{q}_{t} \cdots \mathfrak{q}_{s} \mathfrak{q}_{s+1}^{d-s-1}\right)^{n}\right) T^{n} \rightarrow 0
$$

with respect to $x_{1}, \ldots, x_{t-1}$, we obtain that

$$
H^{p}\left(x_{1}, \ldots, x_{t-1} ; R\left(\mathfrak{q}_{t} \cdots \mathfrak{q}_{s} \mathfrak{q}_{s+1}^{d-s-1}\right)\right)=0 \quad \text { for } p<t-1
$$

and

$$
H^{t-1}\left(x_{1}, \ldots, x_{t-1} ; R\left(\mathfrak{q}_{t} \cdots \mathfrak{q}_{s} \mathfrak{q}_{s+1}^{d-s-1}\right)\right) \cong R\left(\mathfrak{q}_{t} \cdots \mathfrak{q}_{s} \mathfrak{q}_{s+1}^{d-s-1} \bar{A}\right)
$$

That is, $x_{1}, \ldots, x_{t-1}$ is a regular sequence on $R\left(\mathfrak{q}_{t} \cdots \mathfrak{q}_{s} \mathfrak{q}_{s+1}^{d-s-1}\right)$ and

$$
R\left(\mathfrak{q}_{t} \cdots \mathfrak{q}_{s} \mathfrak{q}_{s+1}^{d-s-1} \bar{A}\right) \cong R\left(\mathfrak{q}_{t} \cdots \mathfrak{q}_{s} \mathfrak{q}_{s+1}^{d-s-1}\right) /\left(x_{1}, \ldots, x_{t-1}\right) R\left(\mathfrak{q}_{t} \cdots \mathfrak{q}_{s} \mathfrak{q}_{s+1}^{d-s-1}\right) .
$$

Therefore $R\left(\mathfrak{q}_{t} \cdots \mathfrak{q}_{s} \mathfrak{q}_{s+1}^{d-s-1}\right)$ is a Cohen-Macaulay ring.

Proof of Theorem 1.1. Let $A$ be a Noetherian local ring of dimension $d>0$. First we prove that (B) implies (A). Assume that $A$ satisfies (B). If $d=1$, then $A$ is Cohen-Macaulay because $A$ has no embedded prime. Let $a$ be a system of parameters for $A$. Then $R(a A)$ is a polynomial ring over $A$ and hence CohenMacaulay.

Assume that $d \geq 2$. Since $A$ is unmixed, $\operatorname{dim} A / \mathfrak{p}=d$ for any associated prime $\mathfrak{p}$ of $A$. Thus $s=\operatorname{dim} A / \mathfrak{a}(A)<d-1$ because of Corollary 2.4 Theorem 2.5 assures us that there exists a $p$-standard system of parameters $x_{1}, \ldots, x_{d}$ of type $s$ for $A$. Since $A$ is unmixed, $x_{1}, \ldots, x_{d}$ are non-zero divisors on $A$. Therefore Corollary 4.5 gives an arithmetic Macaulayfication of $A$.

Next we show that $(\mathrm{A})$ implies $(\mathrm{B})$. Let $\mathfrak{b}$ be an ideal in $A$ of positive height such that $R=A[\mathfrak{b} T]$ is a Cohen-Macaulay ring. Then $A$ is a homomorphic image of a Cohen-Macaulay local ring $R_{\mathfrak{m} R+R_{+}}$and hence all the formal fibers of $A$ are CohenMacaulay. Next we show that $A$ is unmixed. By passing through the completion, we may assume that $A$ is complete. Since $\mathfrak{b}$ is of positive height, $\operatorname{dim} R=d+1$. See [32, Corollary 1.6]. Let $\mathfrak{p}_{1}, \ldots, \mathfrak{p}_{s}$ be the associated primes of $A$. Then

$$
\mathfrak{p}_{i}^{*}=\mathfrak{p}_{i} A[T] \cap R \quad \text { where } i=1, \ldots, s
$$


are the associated primes of $R$. Since $R$ is a Cohen-Macaulay ring of dimension $d+1$, $\operatorname{dim} R / \mathfrak{p}_{i}^{*}=d+1$ and hence $\operatorname{dim} A / \mathfrak{p}_{i}=d$; see [32] Corollary 1.6] again, for all $i$.

To close this section, we give an example.

Example 4.6. Let $k$ be a field, $B$ an affine semigroup ring

$$
k\left[a, b, c, d, e^{2}, e^{3}, a d e, b d e, c d e, d^{2} e\right]
$$

and $\mathfrak{n}$ the homogeneous maximal ideal of $B$. Then $A=B_{\mathfrak{n}}$ is a Noetherian local ring of dimension 5 . The sequence $x_{1}=a^{4}, x_{2}=b^{4}, x_{3}=c^{4}, x_{4}=d^{4}, x_{5}=e^{4}$ is a $p$-standard system of parameter of type 3 for $A$. See [17, Appendix B].

Let $\mathfrak{q}_{i}=\left(x_{i}, \ldots, x_{d}\right)$ for $i=1, \ldots, 4$. Then the proof of Corollary 4.5 says that the multi-Rees algebra $A\left[\mathfrak{q}_{1} T_{1}, \ldots, \mathfrak{q}_{4} T_{4}\right]$ is a Cohen-Macaulay ring of dimension 9 . However, we can verify that it is a Cohen-Macaulay ring by using a computer [6]. Indeed the sequence $x_{1}, x_{1} T_{1}+x_{2}, x_{2} T_{1}+x_{3}, x_{2} T_{2}+x_{3} T_{1}+x_{4}, x_{3} T_{2}+x_{4} T_{1}+x_{5}$, $x_{3} T_{3}+x_{4} T_{2}+x_{5} T_{1}, x_{4} T_{3}+x_{5} T_{2}, x_{4} T_{4}+x_{5} T_{3}, x_{5} T_{4}$ is a regular sequence on $A\left[\mathfrak{q}_{1} T_{1}, \ldots, \mathfrak{q}_{4} T_{4}\right]$ of length 9 .

\section{The proof of Corollary 1.2}

Before proving Corollary 1.2 we state the definition of the codimension function.

Definition 5.1. Let $B$ be a Noetherian ring. An integer-valued function $t_{B}$ defined on $\operatorname{Spec} B$ is said to be a codimension function of $B$ if

$$
\text { ht } \mathfrak{p}_{1} / \mathfrak{p}_{2}=t_{B}\left(\mathfrak{p}_{1}\right)-t_{B}\left(\mathfrak{p}_{2}\right) \quad \text { whenever } \mathfrak{p}_{1} \supseteq \mathfrak{p}_{2} .
$$

A codimension function of $B$ is not unique even if it exists. In fact, if $t(\mathfrak{p})$ is a codimension function, then $t(\mathfrak{p})+c$ is also a codimension function for any constant $c$. However, the codimension function is unique up to constant if $\operatorname{Spec} B$ is connected.

Proposition 5.2. (1) A catenary local ring has a codimension function.

(2) A catenary integral domain has a codimension function.

(3) A Cohen-Macaulay ring has a codimension function even if it is neither a local ring nor an integral domain.

(4) If a Noetherian ring has a codimension function, then its homomorphic image does also.

(5) If a Noetherian ring has a codimension function, then its localization does also.

(6) A Noetherian ring possessing a dualizing complex has a codimension function.

Proof. Let $B$ be a Noetherian ring.

(1) Let $t(\mathfrak{p})=-\operatorname{dim} B / \mathfrak{p}$. If $B$ is a cantenary local ring, then $t(\mathfrak{p})$ is a codimension function of $B$.

(2) Let $t(\mathfrak{p})=\operatorname{dim} B_{\mathfrak{p}}$. If $B$ is a catenary integral domain, then $t(\mathfrak{p})$ is a codimension function of $B$.

(3) Let $t(\mathfrak{p})=\operatorname{dim} B_{\mathfrak{p}}$. Then $t(\mathfrak{p})$ is the codimension function of $B$. See the proof of [20 Theorem 17.4(ii)].

(4) and (5) Obvious.

(6) See [14, Chapter 5, §7].

A Noetherian ring is catenary if it has a codimension function. But the converse is not necessarily true. Moreover the universally catenarity is independent of the existence of a codimension function. 
Example 5.3. (1) Ogoma [24, §5 I] gave a Noetherian, universally catenary ring with no codimension function.

(2) Nagata [21, Example 2] gave a two-dimensional local integral domain which is not quasi-unmixed. It has a codimension function but is not universally catenary.

If a Noetherian ring $B$ is universally catenary and has a codimension function, then the polynomial ring over $B$ does also.

Theorem 5.4. Let $B$ be a Noetherian, universally catenary ring and $C$ an essentially of finite type $B$-algebra. If $B$ has a codimension function, then $C$ does also.

Proof. We may assume that $C$ is a polynomial ring over $B$. Let $t_{B}$ be a codimension function. We put

$$
t_{C}(\mathfrak{q})=t_{B}(\mathfrak{p})+\text { ht } \mathfrak{q} / \mathfrak{p} C \quad \text { where } \mathfrak{p}=\mathfrak{q} \cap B
$$

for each prime ideal $\mathfrak{q}$ in $C$. Then $t_{C}$ is a codimension function of $C$.

The following is the key lemma for the proof of Corollary 1.2 ,

Lemma 5.5. Let $B$ be a Noetherian, universally categnary ring which has a codimension function. Then it is a homomorphic image of a finite type B-algebra $C$ such that the codimension function of $C$ is a constant on the associated primes of $C$. If, in addition, $B$ is a local ring, then there exists a maximal ideal $\mathfrak{n}$ of $C$ such that $B$ is a homomorphic image of $C_{\mathfrak{n}}$.

Proof. Let $t_{B}$ be a codimension function of $B$ and

$$
(0)=\mathfrak{q}_{1} \cap \cdots \cap \mathfrak{q}_{s}
$$

the irredundant primary decomposition of $(0)$ in $B$. We may assume that

$$
\sup \left\{t_{B}\left(\sqrt{\mathfrak{q}_{i}}\right) \mid i=1, \ldots, s\right\}=0 .
$$

We put $n=-\inf \left\{t_{B}\left(\sqrt{\mathfrak{q}_{i}}\right) \mid i=1, \ldots, s\right\}$ and $n_{i}=-t_{B}\left(\sqrt{\mathfrak{q}_{i}}\right)$ for all $i$. Then

$$
C=B\left[T_{1}, \ldots, T_{n}\right] / \bigcap_{i=1}^{s}\left(\mathfrak{q}_{i}, T_{1}, \ldots, T_{n_{i}}\right) B\left[T_{1}, \ldots, T_{n}\right]
$$

has the required property. If $B$ is a local ring with maximal ideal $\mathfrak{m}$, then $\mathfrak{n}=$ $\mathfrak{m} C+\left(T_{1}, \ldots, T_{n}\right) C$ has the required property.

Proof of Corollary 1.2, The only if part is obvious. We prove the if part. Let $A$ be a Noetherian, universally catenary local ring with maximal ideal $\mathfrak{m}$ and assume that all the formal fibers of $A$ are Cohen-Macaulay. If $\operatorname{dim} A=0$, then $A$ itself is Cohen-Macaulay.

We assume that $\operatorname{dim} A>0$. By modifying the proof of [29, Theorem 5.7], we find that all the formal fibers of an essentially of finite type $A$-algebra are CohenMacaulay. By using this fact and Lemma[5.5, we may assume that $\operatorname{dim} A / \mathfrak{p}=\operatorname{dim} A$ for each associated prime $\mathfrak{p}$ of $A$. It implies that $A$ is unmixed because $A$ is formally catanary and all the formal fibers of $A$ are Cohen-Macaulay. Theorem 1.1 says that there exists an arithmetic Macaulayfication $R$ of $A$. Thus $A$ is a homomorphic image of a Cohen-Macaulay local ring $R_{\mathfrak{m} R+R_{+}}$.

If $A$ is excellent, then any essentially of finite type $A$-algebra is also. Therefore we obtain the second assertion. 
We should mention that Corollary 1.2 is not true for non-local rings. Indeed, all the formal fibers of all the localization of Ogoma's example above are CohenMacaulay. But it is not a homomorphic image of a Cohen-Macaulay ring because it has no codimension function.

\section{NON-LOCAL RINGS}

First we prove Theorem 1.3 Let $B$ be a Noetherian ring with dualizing complex $D$. Then there exists a codimension function $t$ of $B$ such that

$$
H^{p}\left(\operatorname{Hom}_{B}(B / \mathfrak{p}, D)_{\mathfrak{p}}\right)=0 \text { if } p \neq t(\mathfrak{p})
$$

for each prime ideal $\mathfrak{p}$ in $B$. The following lemma is an analogue of Proposition 2.3 and Corollary 2.4 We can prove them by using the local duality theorem. Here ann $M$ denotes the annihilator of a $B$-module $M$.

Lemma 6.1. Let $M$ be a finitely generated $B$-module and $\mathfrak{p}$ a prime ideal in $B$. Assume that $t(\mathfrak{q})=0$ for all minimal prime $\mathfrak{q}$ of $M$. Then $M_{\mathfrak{p}}$ is Cohen-Macaulay if and only if $\mathfrak{p} \nsupseteq \prod_{j>0}$ ann $H^{j}(\operatorname{Hom}(M, D))$.

In particular, if $\mathfrak{p} \supseteq \prod_{j>0}$ ann $H^{j}(\operatorname{Hom}(M, D))$, then $t(\mathfrak{p})>0$. If $t(\mathfrak{q})=0$ for all associated prime $\mathfrak{q}$ of $M$, then $\mathfrak{p} \supseteq \prod_{j>0}$ ann $H^{j}(\operatorname{Hom}(M, D))$ implies that $t(\mathfrak{p}) \geq 2$.

We start the proof of Theorem 1.3 .

Proof of Theorem 1.3, Let $d=\operatorname{dim} B$ and assume that $t(\mathfrak{q})=0$ for all associated primes $\mathfrak{q}$ of $B$. Then $s_{0}=\inf \left\{t(\mathfrak{p}) \mid B_{\mathfrak{p}}\right.$ is not Cohen-Macaulay $\} \geq 2$. If $s$ is an integer such that $d-s_{0} \leq s<d-1$, then there exist elements $x_{1}, \ldots, x_{d}$ in $B$ satisfying the following conditions:

(1) if $\mathfrak{p}$ is a minimal prime of $B /\left(x_{i}, \ldots, x_{d}\right) B$, then $t(\mathfrak{p})=d-i+1$;

(2) $x_{s+1}, \ldots, x_{d} \in \prod_{j>0}$ ann $H^{j}(D)$;

(3) $x_{i} \in \prod_{j>d-i}$ ann $H^{j}\left(\operatorname{Hom}\left(B /\left(x_{i+1}, \ldots, x_{d}\right), D\right)\right)$ for $i \leq s$.

We note that (1) implies $(0): x_{d}=0$. Let $\mathfrak{q}_{i}=\left(x_{i}, \ldots, x_{d}\right)$ for $1 \leq i \leq s+1$ and $R=R\left(\mathfrak{q}_{1} \cdots \mathfrak{q}_{s} \mathfrak{q}_{s+1}^{d-s-1}\right)$.

We show that $R_{\mathfrak{p}}$ is Cohen-Macaulay for all prime ideal $\mathfrak{p}$ in $B$. If $\mathfrak{q}_{1} \cdots \mathfrak{q}_{s+1}^{d-s-1} \not \mathbb{Z}$ $\mathfrak{p}$, then $\prod_{j>0}$ ann $H^{j}(D) \nsubseteq \mathfrak{p}$. Therefore $R_{\mathfrak{p}}$ is a polynomial ring over a CohenMacaulay ring $B_{\mathfrak{p}}$.

Assume that $\mathfrak{q}_{1} \cdots \mathfrak{q}_{s+1}^{d-s-1} \subseteq \mathfrak{p}$. Then $x_{t}, \ldots, x_{d} \in \mathfrak{p}$ and $x_{t-1} \notin \mathfrak{p}$ for some $1 \leq t \leq s+1$, where we put $x_{0}=1$. Taking localization of (1)-(3), we find that

(1) $\operatorname{dim} B_{\mathfrak{p}} /\left(x_{t}, \ldots, x_{d}\right)=\operatorname{dim} B_{\mathfrak{p}}-(d-t+1)$;

(2) $x_{s+1}, \ldots, x_{d} \in \mathfrak{a}\left(B_{\mathfrak{p}}\right)$;

(3) $x_{i} \in \mathfrak{a}\left(B_{\mathfrak{p}} /\left(x_{i+1}, \ldots, x_{d}\right)\right)$ for $t \leq i \leq s+1$;

(4) $\mathfrak{a}\left(B_{\mathfrak{p}} /\left(x_{t}, \ldots, x_{d}\right)\right)=B_{\mathfrak{p}}$ if $t>1$.

Hence $x_{t}, \ldots, x_{d}$ is a subsystem of a $p$-standard system of parameters for $B_{\mathfrak{p}}$ and $B_{\mathfrak{p}} /\left(x_{t}, \ldots, x_{d}\right)$ is Cohen-Macaulay if $t>1$. We find that $R_{\mathfrak{p}}=R\left(\mathfrak{q}_{t} \cdots \mathfrak{q}_{s+1}^{d-s-1} B_{\mathfrak{p}}\right)$ is Cohen-Macaulay by using Corollary 4.5 .

Now Corollary 1.4 becomes trivial.

Proof of Corollary 1.4, Let $B$ be a Noetherian ring with dualizing complex. We may assume that the codimension function of $B$ is a constant on the associated primes of $B$ because of [23, Theorem 3.5]. Then $B$ has an arithmetic Macaulayfication $R$. Since $R$ also has a dualizing complex and is Cohen-Macaulay, $R$ is 
a homomorphic image of a finite-dimensional Gorenstein ring. See 25] and [30. Theorem 4.3]. Therefore $B$ is also.

\section{REFERENCES}

1. Ian M. Aberbach, Arithmetic Macaulayfications using ideals of dimension one, Illinois J. Math. 40 (1996), 518-526. MR 97h:13002

2. Yoichi Aoyama and Shiro Goto, A brief summary of the elements of the theory of dualizing complexes and Sharp's conjecture, The Curve Seminar at Queen's, Vol. 4, Queen's Papers in Pure and Appl. Math., vol. 76, 1986. MR 88g:13013

3. Some special cases of a conjecture of Sharp, J. Math. Kyoto Univ. 26 (1986), 613-634. MR 88h:13013

4. - A conjecture of Sharp - the case of local rings with dimnon-CM $\leq 1$ or $\operatorname{dim} \leq 5$, Algebraic Geometry and Commutative Algebra in Honor of Masayoshi Nagata, Kinokuniya, 1988, pp. 27-34. MR 90b:13018

5. Jacob Barshay, Graded algebras of powers of ideals generated by A-sequences, J. Algebra 25 (1973), 90-99. MR 48:11074

6. Dave Bayer and Michael Stillman, Macaulay: A system for computation in algebraic geometry and commutative algebra, 1982-1994, Source and object code available for Unix and Macintosh computers. Contact the authors, or download from math.harvard.edu via anonymous ftp.

7. Markus Brodmann, Local cohomology of certain Rees- and form-rings I, J. Algebra 81 (1983), 29-57. MR 85b:13030

8. Nguyen Tu Cuong, P-standard systems of parameters and p-standard ideals in local rings, Acta Math. Vietnam. 20 (1995), 145-161. MR 96h:13064

9. Shiro Goto, Blowing-up of Buchsbaum rings, Proceedings, Durham symposium on Commutative Algebra, London Math. Soc. Lect. Notes, vol. 72, Cambridge Univ. Press, 1982, pp. 140162. MR 84h:13032

10. - On the associated graded rings of Buchsbaum rings, J. Algebra 85 (1983), 490-534. MR 85d:13032

11. Shiro Goto and Yasuhiro Shimoda, On Rees algebras over Buchsbaum rings, J. Math. Kyoto Univ. 20 (1980), 691-708. MR 82c:13028

12. Shiro Goto and Kikumichi Yamagishi, The theory of unconditioned strong d-sequences and modules of finite local cohomology, preprint.

13. Alexander Grothendieck, Local cohomology, Lecture Notes in Math., vol. 41, Springer-Verlag, Berlin, Heiderberg, New-York, 1967. MR 37:219

14. Robin Hartshorne, Residue and duality, Lecture Notes in Math., vol. 20, Springer-Verlag, Berlin, Heidelberg, New York, 1966. MR 36:5145

15. Manfred Herrmann, Eero Hyry, and Jürgen Ribbe, On the Cohen-Macaulay and Gorenstein properties of multigraded Rees algebra, Manuscripta Math. 79 (1993), 343-377. MR 94h:13003

16. Eero Hyry, The diagonal subring and the Cohen-Macaulay property of a multigraded ring, Trans. Amer. Math. Soc. 351 (1999), 2213-2232. MR 99i:13005

17. Takesi Kawasaki, On Macaulayfication of Noetherian schemes, Trans. Amer. Math. Soc. 352 (2000), 2517-2552. MR 2000j:14077

18. - On arithmetic Macaulayfication of certain local rings, Comm. Algebra 26 (1998), 4385-4396. MR 99m:13007

19. Kazuhiko Kurano, On Macaulayfication obtained by a blow-up whose center is an equi-multiple ideal, J. Algebra 190 (1997), 405-434, with an appendix by Yamagishi, Kikumich. MR 98f:13004

20. Hideyuki Matsumura, Commutative ring theory, Cambridge Studies in Advanced Math., vol. 8, Cambridge University Press, 1986, First paperback edition, 1989. MR 90i:13001 MR 88h:13001

21. Masayoshi Nagata, Local rings, Interscience Tracts, vol. 13, Interscience, New York-LondonSydney, 1962. MR 27:5790

22. Tetsushi Ogoma, Existence of dualizing complexes, J. Math. Kyoto Univ. 24 (1984), 27-48. MR 85j: 13028

23. Associated primes of fibre product rings and a conjecture of Sharp in lower dimensional cases, Mem. Fac. Sci. Kochi Univ. (Math.) 6 (1985), 1-9. MR 86e:13009 
24. Philos. Soc. 97 (1985), 231-241. MR 86e:13008

25. Idun Reiten, The converse to a theorem of Sharp in Gorenstein modules, Proc. Amer. Math. Soc. 32 (1972), 417-420. MR 45:5128

26. Peter Schenzel, Dualisierende Komplexe in der lokalen Algebra und Buchsbaum-Ringe, Lecture Notes in Math., vol. 907, Springer, Berlin-Heidelberg-New York, 1982. MR 83i:13013

27. $\ldots$ Standard systems of parameters and their blowing-up rings, J. Reine Angew. Math. 344 (1983), 201-220. MR 84m:13025

28. Jean-Pierre Serre, Faisceaux algébriques cohérents, Ann. of Math. (2) 61 (1955), 197-278.

29. Rodney Y. Sharp, Acceptable rings and homomorphic images of Gorenstein rings, J. Algebra 44 (1977), 246-261. MR 56:348

30. - Necessary conditions for the existence of dualizing complexes in commutative algebra, Sém. Algèbre P. Dubreil 1977/78, Lecture Notes in Mathematics, vol. 740, Springer-Verlag, 1979, pp. 213-229. MR 81d:13013

31. Yasuhiro Shimoda, A note on Rees algebras of two dimensional local domains, J. Math. Kyoto Univ. 19 (1979), 327-333. MR 80k:13011

32. Giuseppe Valla, Certain graded algebras are always Cohen-Macaulay, J. Algebra 42 (1976), 537-548. MR 54:10240

Department of Mathematics, Tokyo Metropolitan University, Hachioji-Shi MinamiOHSAWA 1-1, TOKYo 192-0397, JAPAN

E-mail address: kawasaki@comp.metro-u.ac.jp

$U R L:$ http://www. comp.metro-u.ac.jp/ kawasaki/ 